STRUCTURAL BIOLOGY

ISSN 2059-7983

\section{C-phycocyanin as a highly attractive model system in protein crystallography: unique crystallization properties and packing-diversity screening}

\author{
Iosifina Sarrou, ${ }^{\text {a* }}$ Christian G. Feiler, ${ }^{\mathrm{b}}$ Sven Falke, ${ }^{\mathrm{c}, \mathrm{d}}$ Nolan Peard, ${ }^{\mathrm{a}, \mathrm{e}}$ Oleksandr \\ Yefanov $^{\mathrm{a}}$ and Henry Chapman ${ }^{\mathrm{a}, \mathrm{d}, \mathrm{f}}$
}

Received 21 September 2020

Accepted 9 December 2020

Edited by J. Newman, Bio21 Collaborative Crystallisation Centre, Australia

Keywords: C-phycocyanin; crystal packing; model system; precipitant; crystallization determinants.

PDB references: C-phycocyanin, space group $P 6_{3}, 1.45 \AA$ resolution, 6yqg; $1.8 \AA$ 政olution, 6yq8; space group $R 32,1.29 \AA$ resolution, 6ypq; space group $P 22_{1} 22,2.1 \AA$ resolution, 6yyj

Supporting information: this article has supporting information at journals.iucr.org/d

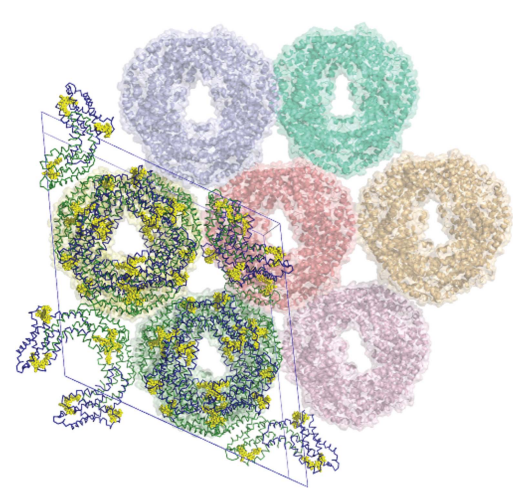
OPEN ○ ACCESS
${ }^{\mathbf{a} C}$ Centre for Free-Electron Laser Science, DESY, Notkestrasse 85, 22607 Hamburg, Germany, ${ }^{\mathbf{b}}$ Helmholtz-Zentrum Berlin Infection and Inflammation, Universität Hamburg, Notkestrasse 85, 22607 Hamburg, Germany, ${ }^{\mathbf{d} H a m b u r g}$ Centre for Ultrafast Imaging, Universität Hamburg, Luruper Chaussee 149, 22607 Hamburg, Germany, 'epepartment of Physics, Massachusetts Institute of Technology, 77 Massachusetts Avenue, Cambridge, MA 02139, USA, and 'Department of Physics, Universität Hamburg, Luruper Chaussee 149, 22607 Hamburg, Germany. ${ }^{*}$ Correspondence e-mail: iosifina.sarrou@cfel.de

The unique crystallization properties of the antenna protein C-phycocyanin (C-PC) from the thermophilic cyanobacterium Thermosynechococcus elongatus are reported and discussed. C-PC crystallizes in hundreds of significantly different conditions within a broad $\mathrm{pH}$ range and in the presence of a wide variety of precipitants and additives. Remarkably, the crystal dimensions vary from a few micrometres, as used in serial crystallography, to several hundred micrometres, with a very diverse crystal morphology. More than 100 unique single-crystal X-ray diffraction data sets were collected from randomly selected crystals and analysed. The addition of small-molecule additives revealed three new crystal packings of C-PC, which are discussed in detail. The high propensity of this protein to crystallize, combined with its natural blue colour and its fluorescence characteristics, make it an excellent candidate as a superior and highly adaptable model system in crystallography. C-PC can be used in technical and methods development approaches for X-ray and neutron diffraction techniques, and as a system for comprehending the fundamental principles of protein crystallography.

\section{Introduction}

Protein X-ray crystallographic techniques have been used extensively to determine static macromolecular structures and also to analyse protein dynamics (Liebschner, 2018; Spence, 2017). Hence, high-quality protein crystals are necessary to achieve electron-density maps of high resolution and high confidence, which allow the study of molecular mechanisms and of the function and interactions of biomacromolecules (Liebschner, 2018; Chayen, 2009).

Protein crystallization is a multi-parametric process and depends on several factors, including protein concentration, sample purity, temperature, $\mathrm{pH}$ value, precipitant, buffers, additives, detergents, force fields and pressure, which can be visualized in multi-dimensional phase diagrams (Chayen et al., 1992; Chayen, 2009; Rupp, 2010; McPherson \& Cudney, 2006). Protein crystal nucleation is described as a multi-step process with the early formation of a dense liquid protein phase, which precedes the growth of a rigid nucleus (Sauter et al., 2015).

The most popular approach to initial crystallization trials applies sparse-matrix screens of 96 different conditions at a time, which are subsequently refined according to the successful hits (Chayen, 2009; Rupp, 2010). However, it is challenging to identify those crystallization conditions that für Materialien und Energie, Albert-Einstein-Strasse 15, 12489 Berlin, Germany, ' Laboratory for Structural Biology of 
yield high-quality diffracting crystals (Chayen \& Saridakis, 2008). It is not feasible to make a prediction, based on the chemical and physical properties of a protein, of the conditions required to crystallize it, even with efforts to monitor and actively influence the crystallization process (Falke \& Betzel, 2019). Changes in a single experimental parameter can simultaneously affect several aspects of a crystallization experiment (Chayen, 2009). Many efforts, over decades, have aimed at improving the success of crystallization experiments and the resulting crystal size (Rupp, 2010; McPherson \& Cudney, 2006). However, the success of crystallization methods widely depends on trial and error (DePristo et al., 2004; Groot et al., 1998).

Moreover, methods development in macromolecular X-ray crystallography often depends upon the use of easy-to-handle proteins that are highly stable and available in bulk amounts, such as insulin, proteinase $\mathrm{K}$ and hen egg-white lysozyme. The crystallization abilities of the latter protein have been well studied, involving different space groups, crystal morphologies and sizes for the development of multiple applications (Panjikar et al., 2015; Meents et al., 2017; Haas, 2020).

This study focuses on examining the robust crystallization of C-phycocyanin (C-PC), which is almost independent of the composition of the crystallization solution, and investigating the resulting crystal morphology, molecular packing and crystal quality. Several crystal structures of the cyanobacterial antenna protein C-PC (Nield et al., 2003; Adir et al., 2002) have already been reported and information on selected C-PC structures from various cyanobacteria is shown in Supplementary Table S1.

This study demonstrates that C-PC from Thermosynechococcus elongatus, purified using a highly efficient one-column purification protocol, can be crystallized using hundreds of diverse crystallization solutions in numerous crystal symmetries at different $\mathrm{pH}$ values and with additives. Various crystal morphologies can be recognized, from which several dozen high-resolution X-ray diffraction data sets have been collected. Different crystal packings were observed when small drug-like molecules were present in the crystallization solutions. An in-depth analysis of these molecular assemblies will be helpful in comprehending the uncommon crystallization behaviour of native C-PC.

The antenna protein C-PC appears to be an essential sample for methods development in protein crystallography. In combination with their optical properties, blue colour and strong fluorescence, C-PC crystals may inspire new applications and studies in biomolecular crystallography.

\section{Materials and methods}

\subsection{Purification, characterization in solution and} crystallization of C-PC

C-PC from T. elongatus was isolated based on a protocol previously described elsewhere (Nield et al., 2003) with an additional chromatography step using a DEAE chromatography column equilibrated with $20 \mathrm{~m} M$ MES pH 6.5 or $20 \mathrm{~m} M$ Tris $\mathrm{pH} 8.0$ (depending on the downstream crystal- lization experiment) and mounted on an ÄKTA purifier (GE Healthcare, USA). Every litre of cell culture produces $1 \mathrm{~g}$ of wet cells, resulting in approximately $8 \mathrm{mg}$ purified C-PC.

When studied at $\mathrm{pH} 4.0$, the protein was dialyzed against $20 \mathrm{~m} M$ sodium acetate, $100 \mathrm{mM} \mathrm{NaCl}$.

$\mathrm{CD}$ spectroscopy experiments were performed to verify the overall folding and secondary-structure composition of C-PC in different buffers using a Jasco J-810 spectrometer (Jasco, UK). Spectra of pure C-PC $\left(0.1 \mathrm{mg} \mathrm{ml}^{-1}\right)$ were recorded in the far-UV wavelength range between 195 and $240 \mathrm{~nm}$ at $20^{\circ} \mathrm{C}$ using a $1 \mathrm{~mm}$ path-length quartz cell with a scanning speed of $100 \mathrm{~nm} \mathrm{~min}{ }^{-1}$. The obtained spectra provide a fingerprint of the secondary-structure composition of C-PC. Ellipticity values were scaled and provided as the mean molar ellipticity (MME). Ten spectra were averaged.

The dispersity and particle-size distribution of the protein in solutions at different $\mathrm{pH}$ values was verified via infrared dynamic light scattering (IR-DLS) using a DynaPro NanoStar instrument (Wyatt Instruments, USA) equipped with a $785 \mathrm{~nm}$ wavelength laser. C-PC naturally has a strong absorption maximum at approximately $600 \mathrm{~nm}$ and fluorescence at about $640 \mathrm{~nm}$. The optical properties of C-PC are taken into account when the protein is studied in solution and the crystalline form.

Prior to crystallization, C-PC was dialyzed against the respective chromatography buffer supplemented with $100 \mathrm{mM}$ $\mathrm{NaCl}$. The protein buffer concentration was limited to $20 \mathrm{mM}$ in order to maximize the effect of extreme $\mathrm{pH}$ values of the crystallization solutions after mixing.

The protein was concentrated using Amicon Centricon YM-10 centrifugal filters at $1500 \mathrm{~g}$ to a final concentration of $15 \mathrm{mg} \mathrm{ml}^{-1}$.

The crystallization experiments were set up manually in 96-well plates (MRC 2-well; Jena Bioscience) using the commercially available screens JCSG-plus, Morpheus, Morpheus 3, Pi-Minimal, PACT, SG1, PGA and Midas from Molecular Dimensions and Jena Bioscience. In each sittingdrop vapour-diffusion experiment, $1 \mu \mathrm{l}$ protein solution was mixed with $1 \mu \mathrm{l}$ reservoir solution and incubated against $50 \mu \mathrm{l}$ reservoir solution. Crystallization plates were incubated at $22^{\circ} \mathrm{C}$ and automatically imaged by second-order nonlinear imaging of chiral crystals (SONICC; Formulatrix, Bedford, Massachusetts, USA).

The presence of crystalline material $(>1 \mu \mathrm{m})$ was verified in each crystallization drop using SONICC. This secondharmonic generation (SHG) imaging relies on a nonlinear optical process of frequency doubling in chiral non-centrosymmetric crystals to provide information on the crystallinity (Boyd, 2008). The UV-TPEF (ultraviolet two-photon excited fluorescence) method is analogous to classical UV fluorescence and generates images based on the fluorescence of UVexcited aromatic amino acids.

All crystals obtained grew to full size within two days or less, with various sizes and different shapes. Unless the crystallization condition contained a cryoprotectant, crystals were briefly washed with a cryoprotectant solution containing the mother liquor supplemented with 25\% PEG 400 before flashcooling in liquid nitrogen. 
Table 1

Data collection and processing.

Values in parentheses are for the outer shell.

\begin{tabular}{|c|c|c|c|c|}
\hline Structure & C-PC, $P 6_{3}$-large & C-PC, $P 6_{3}$-small & C-PC, $R 32$ & C-PC, $P 2{ }_{1} 2{ }_{1} 2$ \\
\hline Diffraction source & P11, PETRA III, DESY & P11, PETRA III, DESY & P11, PETRA III, DESY & P11, PETRA III, DESY \\
\hline Wavelength $(\AA)$ & 1.0332 & 1.0332 & 1.0332 & 1.0332 \\
\hline Temperature (K) & 100 & 100 & 100 & 100 \\
\hline Detector & PILATUS3 S 6M & PILATUS3 S 6M & PILATUS3 S 6M & PILATUS3 S 6M \\
\hline Crystal-to-detector distance (mm) & 300 & 160 & 225 & 160 \\
\hline Rotation range per image $\left(^{\circ}\right)$ & 0.1 & 0.1 & 0.1 & 0.2 \\
\hline Total rotation range $\left({ }^{\circ}\right)$ & 200 & 200 & 180 & 100 \\
\hline Exposure time per image (s) & 0.05 & 0.05 & 0.05 & 0.05 \\
\hline Space group & $P 6_{3}$ & $P 6_{3}$ & $R 32$ & $P 2_{1} 2_{1} 2$ \\
\hline$a, b, c(\AA)$ & $153.51,153.51,39.36$ & $108.20,108.20,66.05$ & $187.17,187.17,59.93$ & $118.36,98.23,104.6$ \\
\hline$\alpha, \beta, \gamma\left({ }^{\circ}\right)$ & $90,90,120$ & $90,90,120$ & $90,90,120$ & $90,90,90$ \\
\hline Mosaicity $\left({ }^{\circ}\right)$ & 0.103 & 0.078 & 0.05 & 0.23 \\
\hline Resolution range $(\AA)$ & $44.32-1.82(1.88-1.82)$ & $46.86-1.45(1.50-1.45)$ & $48.19-1.29(1.33-1.29)$ & $47.84-2.16(2.23-2.16)$ \\
\hline Total No. of reflections & 526799 & 439602 & 890243 & 488033 \\
\hline No. of unique reflections & $48123(4751)$ & $77962(7750)$ & 99231 (9099) & $65961(6460)$ \\
\hline Completeness (\%) & $99.9(99.8)$ & $99.9(99.8)$ & $99.0(91.3)$ & $99.4(96.8)$ \\
\hline Multiplicity & 11.13 & 5.73 & 8.97 & 7.41 \\
\hline$\langle I / \sigma(I)\rangle$ & $22.2(0.82)$ & $9.95(0.53)$ & $31.44(4.44)$ & $7.41(0.69)$ \\
\hline$R_{\text {r.i.m. }}(\%)$ & $6.1(267)$ & $9.6(98.8)$ & $3.7(39.5)$ & $26.1(292)$ \\
\hline Overall $B$ factor from Wilson plot $\left(\AA^{2}\right)$ & 41.33 & 23.60 & 14.33 & 38.62 \\
\hline
\end{tabular}

\subsection{Data collection}

Diffraction data were collected from single crystals on beamline P11 at the PETRA III electron-storage ring, DESY, Hamburg, using a PILATUS3 S 6M detector. All data were collected as non-overlapping $0.1^{\circ}$ oscillation images, indexed and integrated with XDSAPP (Sparta et al., 2016), and scaled with AIMLESS from the CCP4 suite (Winn et al., 2011). A statistically significant value for $\mathrm{CC}_{1 / 2}$ (Karplus \& Diederichs, 2012) in the highest resolution shell was chosen as a cutoff criterion respecting the completeness of the data. Indexing parameters are summarized in Supplementary Tables S2 and S3. Collected raw diffraction images are publicly available via https://proteindiffraction.org/.

\subsection{Structure determination and refinement}

The crystal structures of C-PC were determined by molecular replacement with phenix.phaser (Liebschner et al., 2019) using a heterodimer extracted from PDB entry 1jbo (Nield et $a l ., 2003)$ as a search model. Iterative automated refinement was carried out with phenix.refine (Liebschner et al., 2019), and manual adjustments and model optimization were performed by hand in Coot (Emsley et al., 2010). Structural coordinates have been deposited in the Protein Data Bank with accession codes 6yyj (space group $P 2_{1} 2_{1} 2$ ), $6 y q 8$ (space group $P 6_{3}$, larger unit cell), 6yqg (space group $P 6_{3}$, smaller unit cell) and 6ypq (space group R32). Data-collection and refinement statistics are also summarized in Tables 1 and 2.

\section{Results and discussion}

\subsection{Purification of C-PC and characterization in solution}

Before the crystallization experiments, C-PC was purified to homogeneity and characterized in solution using CD spectroscopy to verify the folding and DLS to investigate the solution dispersity (Fig. 1). As observed using IR-DLS measurements, prior to crystallization, with $\mathrm{C}-\mathrm{PC}$ at $\mathrm{pH} 4.0$, 6.5 and 8.0, the hydrodynamic radius is significantly increased at $\mathrm{pH} 4.0$ with increased polydispersity. C-PC in solution at $\mathrm{pH}$ 8.0, 6.5 and 4.0 showed hydrodynamic radii of $4.2 \mathrm{~nm}(12 \%$ polydispersity), $4.8 \mathrm{~nm}$ (32\% polydispersity) and $6.3 \mathrm{~nm}(40 \%$ polydispersity), respectively.

Interestingly, after $72 \mathrm{~h}$, in the protein solution at $\mathrm{pH} 4.0$ particles with lattice order and diameters in the range 4-20 $\mu \mathrm{m}$ appeared; this was not the case for the protein at $\mathrm{pH} 6.5$ and $\mathrm{pH}$ 8.0. These micrometre-sized particles were indeed identified as crystalline material. Diffraction data of self-assembled crystals were collected by a serial crystallography approach using a porous polyimide support (Feiler et al., 2019; Supplementary Figs. S1c and S1d), the results will be published elsewhere in more detail. Therefore, we conclude that lower $\mathrm{pH}$ values promote the crystallization of C-PC via autoassembly. The higher percentage of crystallization conditions providing crystals when the protein was buffered at $\mathrm{pH} 6.5$ instead of $\mathrm{pH} 8.0$ would be consistent with these results. The particular reasons for the self-assembly of C-PC towards crystalline particles at lower $\mathrm{pH}$ are still under investigation and might be connected to the in vivo function of C-PC, since phycobilosomes are naturally organized into rods attached to the thylakoid membrane in cyanobacteria and algae (Blankenship, 2015).

Based on these results, crystallization setups were focused on using C-PC buffered at $\mathrm{pH} 8.0$ and $\mathrm{pH} 6.5$.

\subsection{Crystallization experiments}

The crystallization plates were automatically imaged at $22^{\circ} \mathrm{C}$ and two types of image data were recorded: visible and SHG (Supplementary Figs. S3 and S4). A numerical summary of the imaging results depending on the screen is shown in Table 3 . The values refer to the total number of conditions 
Table 2

Structure solution and refinement.

Values in parentheses are for the outer shell.

\begin{tabular}{|c|c|c|c|c|}
\hline Structure name & $\mathrm{C}$-PC, $P 6_{3}$-large & $\mathrm{C}$-PC, $P 6_{3}$-small & $\mathrm{C}-\mathrm{PC}, R 32$ & $\mathrm{C}-\mathrm{PC}, P 2_{1} 2_{1} 2$ \\
\hline Resolution range $(\AA)$ & $44.31-1.82(1.88-1.82)$ & $46.85-1.45(1.50-1.45)$ & $46.79-1.29(1.33-1.29)$ & $47.84-2.16(2.23-2.16)$ \\
\hline Completeness (\%) & $99.85(98.6)$ & $99.7(99.0)$ & $99.0(91.3)$ & $99.5(95.2)$ \\
\hline No. of reflections, working set & $48121(4751)$ & 77950 (7749) & $99198(9095)$ & 65951 (6449) \\
\hline Final $R_{\text {cryst }}$ & $0.179(0.35)$ & $0.178(0.483)$ & $0.153(0.244)$ & $0.204(0.328)$ \\
\hline Final $R_{\text {free }}$ & $0.201(0.40)$ & $0.208(0.475)$ & $0.170(0.257)$ & $0.258(0.393)$ \\
\hline \multicolumn{5}{|l|}{ No. of non-H atoms } \\
\hline Total & 2960 & 3081 & 3160 & 8158 \\
\hline \multicolumn{5}{|l|}{ R.m.s. deviations } \\
\hline Bonds $(\AA)$ & 0.013 & 0.006 & 0.007 & 0.013 \\
\hline Angles $\left({ }^{\circ}\right)$ & 1.07 & 0.98 & 0.89 & 1.5 \\
\hline \multicolumn{5}{|l|}{ Average $B$ factors $\left(\AA^{2}\right)$} \\
\hline Overall & 59.0 & 32.84 & 23.55 & 44.9 \\
\hline Allowed (\%) & 1.5 & 1.83 & 1.53 & 2.75 \\
\hline
\end{tabular}

with visible crystals or microcrystals which showed SHG signal. To avoid false positives, plates were imaged by UVTPEF to confirm that the SHG-positive crystals are indeed protein crystals, especially in the case of microcrystals (Supplementary Fig. S2). Since the laser illumination for the imaging causes damage to the protein crystals owing to local heating, the exposure was limited to the default value in order to proceed to X-ray single-crystal experiments.

C-PC in MES buffer $\mathrm{pH} 6.5$ was screened using eight commercially available screens (Table 3), i.e. testing a total number of 786 different crystallization conditions. After $48 \mathrm{~h}$, crystals could be detected in 724 crystallization conditions covering $92 \%$ of all conditions tested. For comparison, C-PC in Tris buffer $\mathrm{pH} 8.0$ was screened against four commercial screens (Table 3), i.e. 384 individual conditions were tested. After two days, the plates were inspected and crystals could be detected in 291 conditions that were cross-verified with the SHG signal. All of the imaging results under visible light and SHG of the 96-well plates are shown in Supplementary Figs. S3 and S4.

In total, this experiment exhibited protein crystals in more than 1000 different conditions, which appeared in different morphologies and sizes. The details of the statistics for the eight screens with C-PC at pH 6.5 are shown in Fig. 2. While most of the conditions foster the formation of crystals in general, the space group, maximum resolution and other

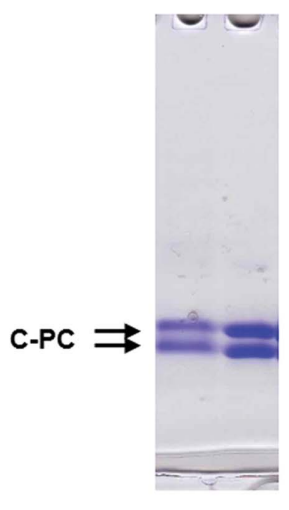

(a)

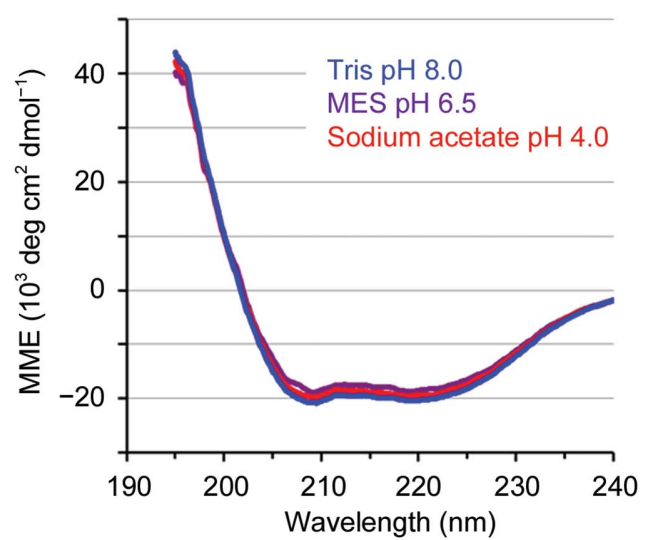

(b)

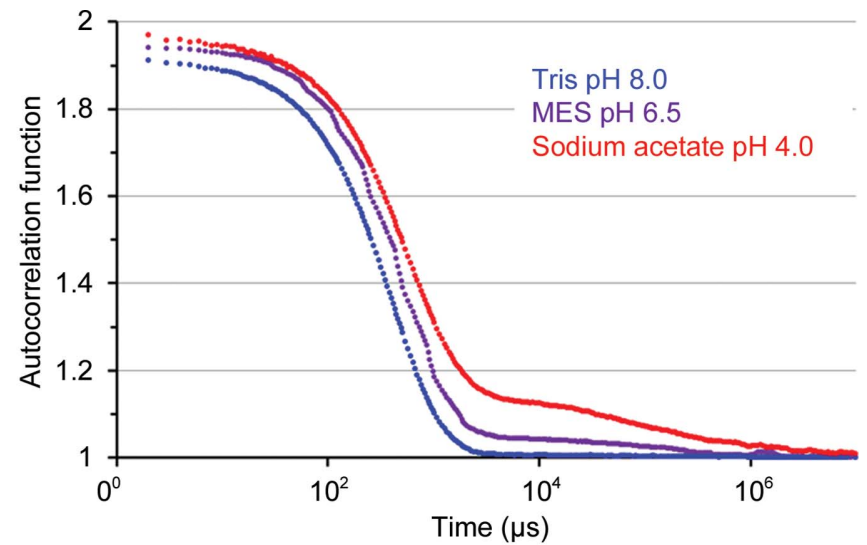

(c)

Figure 1

Characterization of C-PC sample solutions prior to crystallization. (a) SDS-PAGE (12\% acrylamide) analysis of the chromatographic fractions containing pure C-PC used in crystallization trials. (b) Averaged spectra of C-PC obtained by CD spectroscopy buffered at $\mathrm{pH} 8.0$ (20 mM Tris; blue), pH 6.5 (20 mM MES; purple) and pH 4.0 (20 mM acetate; red), indicating a nearly identical secondary structure in all three buffers. (c) Autocorrelation functions of C-PC depending on the delay time as obtained by IR-DLS indicate an increased degree of C-PC solution polydispersity and additional larger particles at $\mathrm{pH} 4.0$ compared with $\mathrm{pH} 8.0$ and $\mathrm{pH} 6.5$. 
Table 3

The number of conditions in which crystals appeared after $48 \mathrm{~h}$.

SONICC and transmitted light microscope images of the 96-well plates are shown in Supplementary Figs. S3 and S4.

\begin{tabular}{lll}
\hline & \multicolumn{2}{l}{ No. of hits per 96 conditions } \\
\cline { 2 - 3 } Crystallization screen & C-PC at $\mathrm{pH} 6.5$ & C-PC at $\mathrm{pH} 8.0$ \\
\hline JCSG-plus & $82 / 96$ & $76 / 96$ \\
Morpheus & $90 / 96$ & $\dagger$ \\
Morpheus 3 & $95 / 96$ & $63 / 96$ \\
Pi-Minimal & $88 / 96$ & $\dagger$ \\
PACT & $96 / 96$ & $\dagger$ \\
SG11 & $94 / 96$ & $\dagger$ \\
PGA & $96 / 96$ & $90 / 96$ \\
Midas & $83 / 96$ & $62 / 96$ \\
\hline
\end{tabular}

$\dagger$ Not determined.

parameters depend on the individual compositions of the crystallization solution. Furthermore, the screens show large differences in the morphologies and sizes of the crystals. For example, in the PACT screen, which contains PEG in all conditions, there is a majority of hits with hexagonal crystals over hits with needle- or feather-like shapes. However, the different crystal morphologies could neither be correlated with the $\mathrm{pH}$ value of the crystallization condition nor with the precipitant. It has not been possible to correlate a specific chemical component with a specific morphology, as attempted in other cases (He et al., 2020), as several widely different conditions result in the same morphology. On the other hand, one specific crystallization solution (e.g. PGA condition B9, Midas condition C6) can also result in a mixture of crystals with significantly different morphology in the same droplet but, as far as we analyzed, with the same space group and molecular packing. Therefore, in contrast to other cases (Frey et al., 1991), the morphology also does not seem to indicate an individual space group and packing. For some conditions, however, we saw that crystals with different morphologies appeared in the same droplet after significantly different incubation times. This might lead to the very general assumption that the morphology is determined by a combination of the initial crystallization solution composition, which determines, for example, a specific second virial coefficient, and the growth speed, which is affected by the local protein concentration at a specific time of incubation.

These unique properties of the crystal formation of C-PC will provide valuable information for future studies of protein crystallogenesis (Lorber, 2005). It is worth mentioning that similar morphologies with size variations and a dependency on the screen were observed in the trays where the protein was purified at $\mathrm{pH} 8.0$ (Supplementary Fig. S5). Examples portraying the different crystal morphologies observed at $20^{\circ} \mathrm{C}$ are shown in Fig. 3 .

\subsection{Data collection}

A few hundred crystals with sizes larger than $70 \mu \mathrm{m}$ were picked and cryocooled in liquid nitrogen after incubation for a few seconds in a cryoprotectant solution that consisted of the mother liquor supplemented with cryoprotectant when necessary. The crystals were selected from 12 screens, as shown in Table 3. We checked more than 200 different crystals for diffraction. Table 4 contains examples of the diverse crystallization conditions which resulted in crystals that diffracted to high resolution. With various combinations of precipitants and $\mathrm{pH}$ values, C-PC produces crystals with the same symmetry. Table 5 summarizes the properties of 118 individually collected data sets. The outcome of the datacollection analysis is presented in Supplementary Tables S2 and S3.

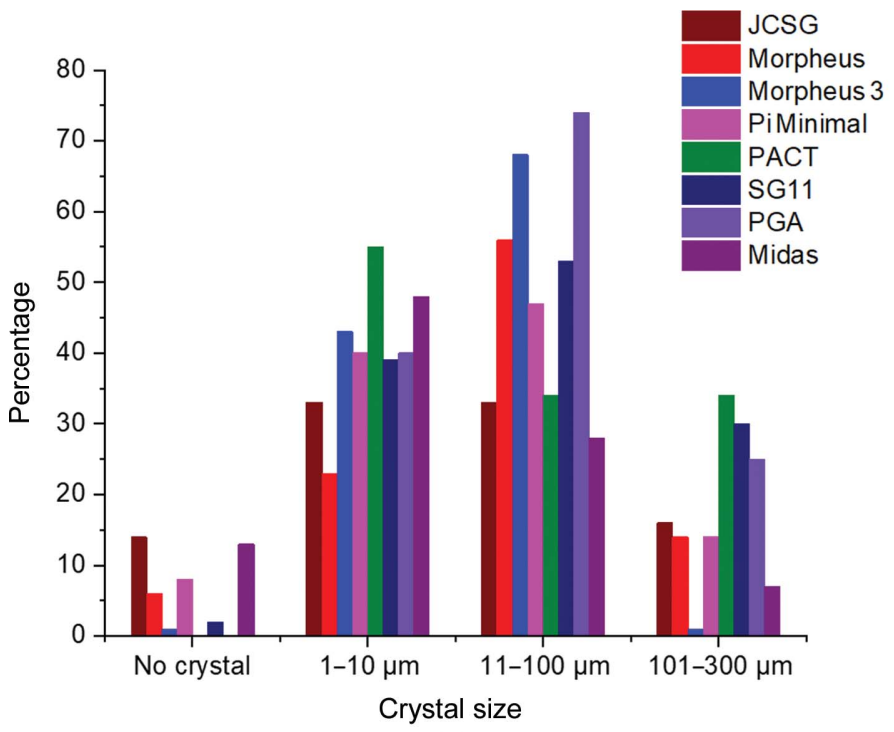

(a)

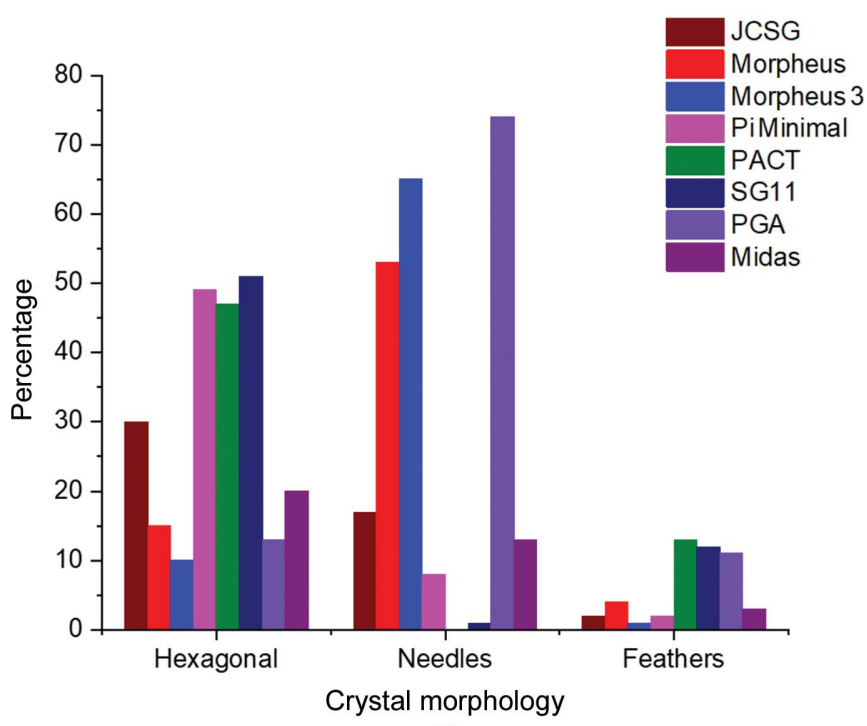

(b)

Figure 2

(a) The statistics of crystal size as they appear under different conditions. Naturally, crystal sizes may vary within one crystallization drop; the results are determined based on the majority of crystals. In some cases, where the crystals have two distinct representative size regimes, both are included; see the examples in Supplementary Fig. S2. (b) Three categories of crystal morphology in the C-PC crystallization experiments utilizing eight different screens as shown in Table 3, with C-PC in MES buffer at $\mathrm{pH}$ 6.5. Please note that the morphologies are reported for crystals larger than $10 \mu \mathrm{m}$. 

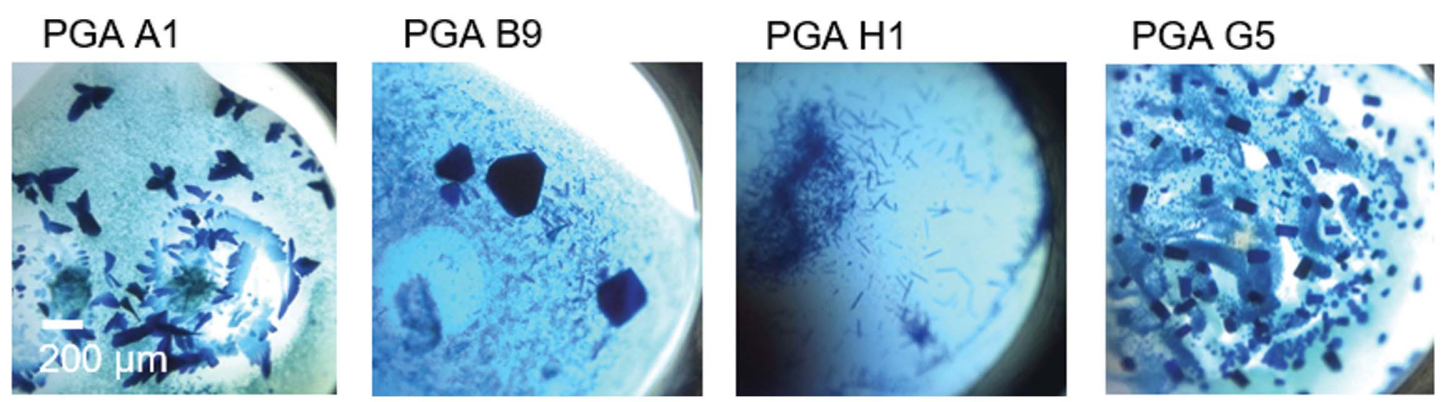

Morpheus A3
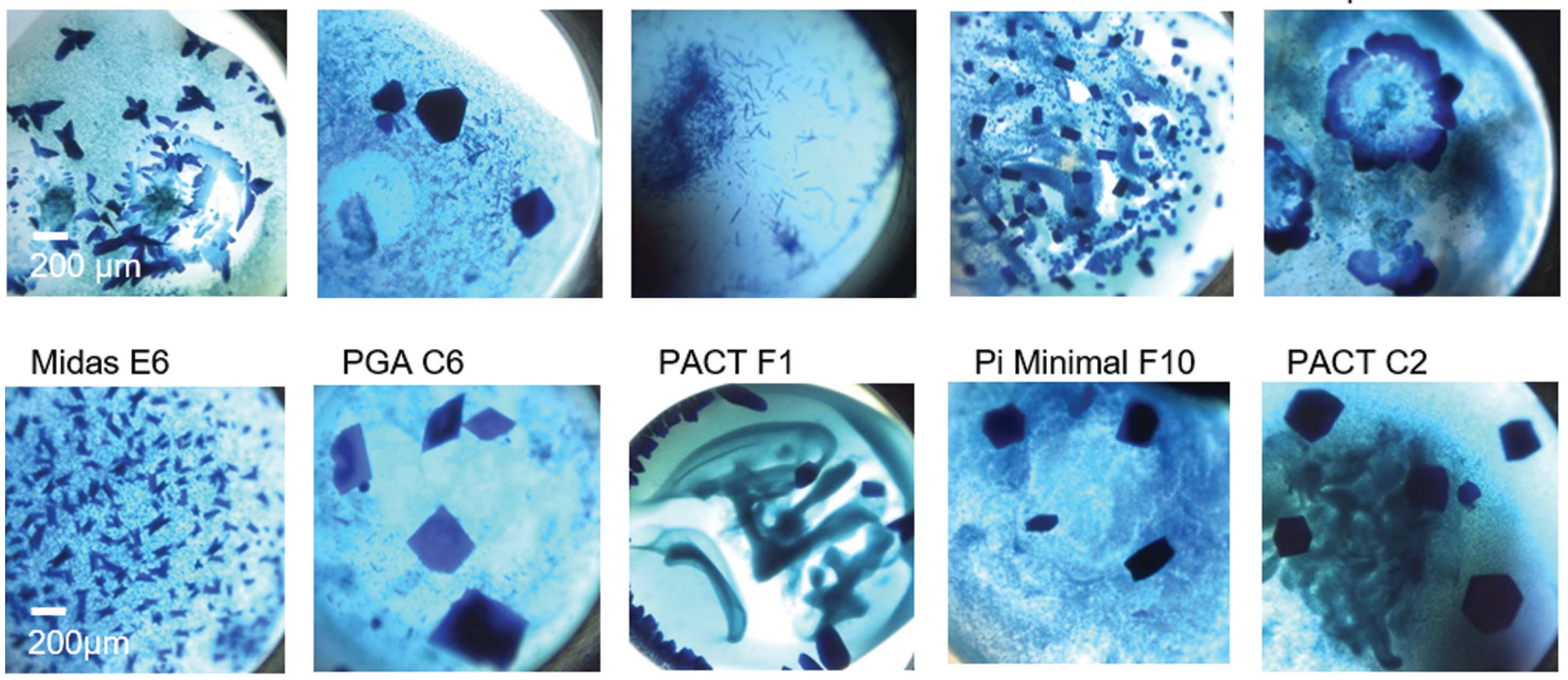
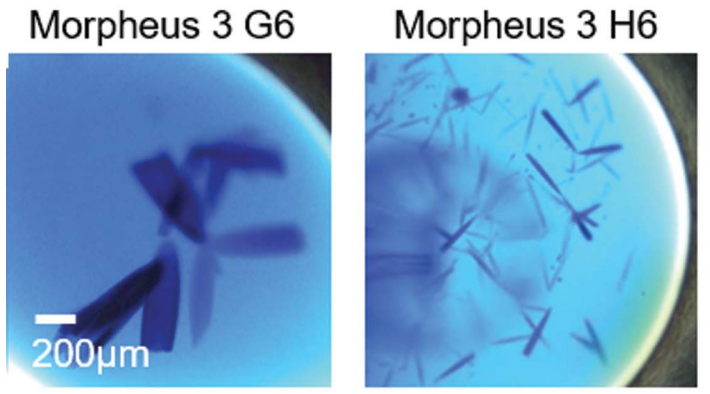

Morpheus 3 F1

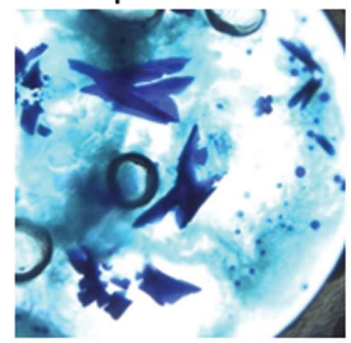

Pi Minimal C4
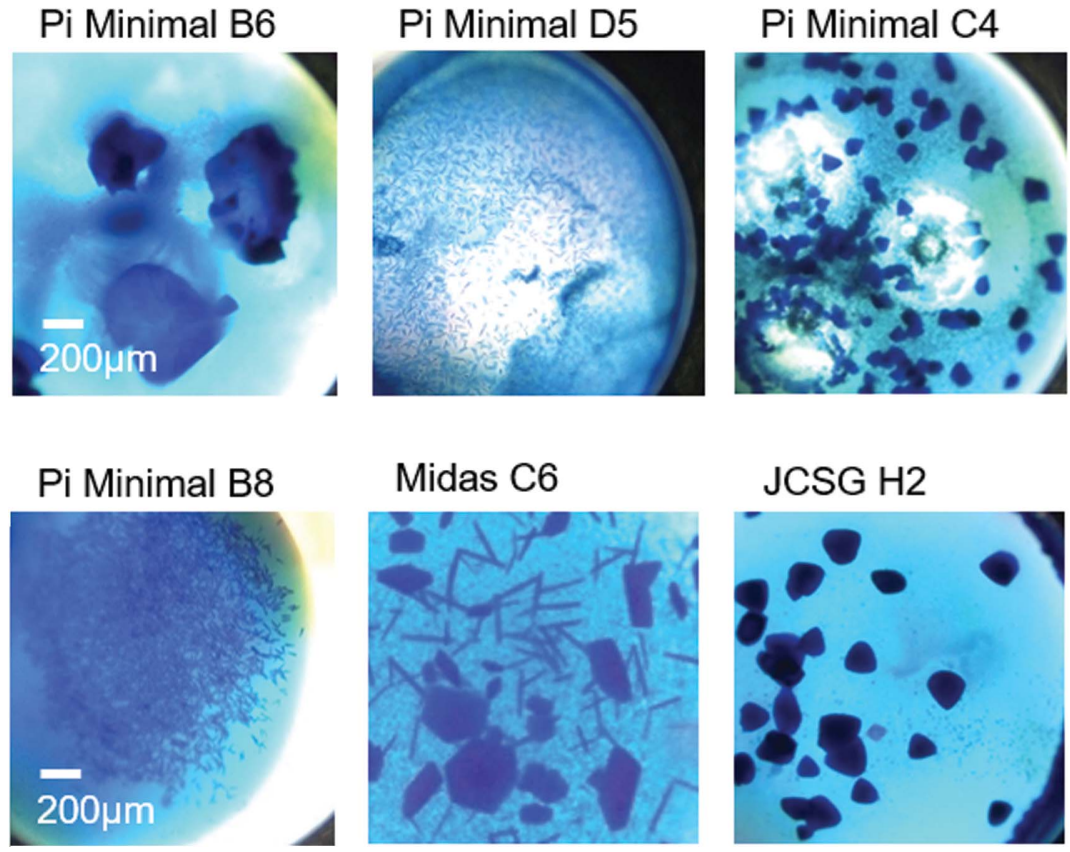

Midas C6

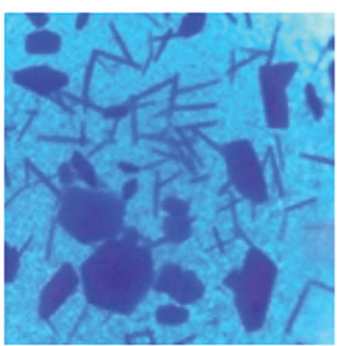

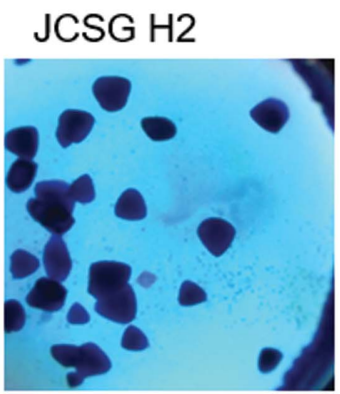

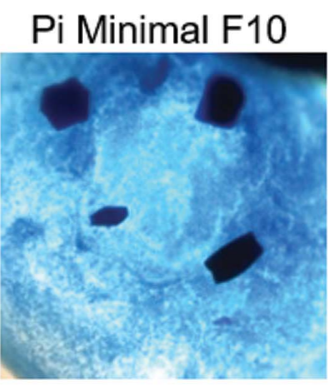

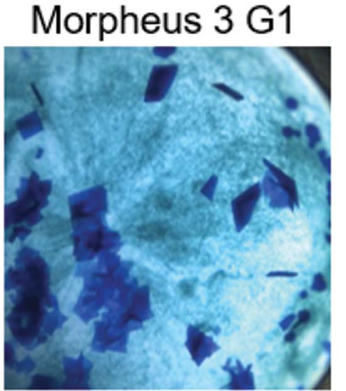
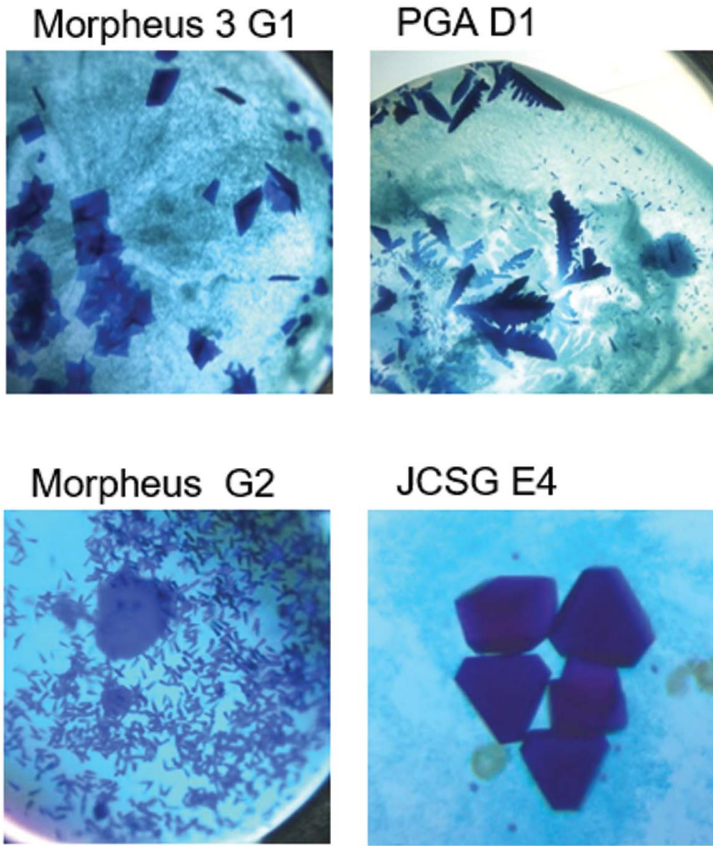

JCSG E4

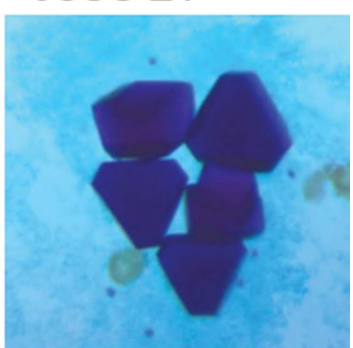

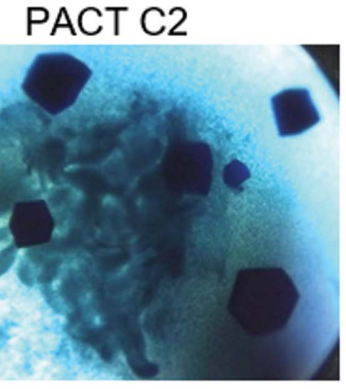
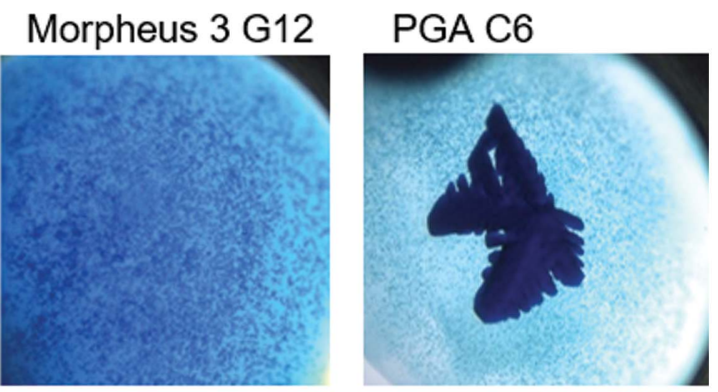

Figure 3

C-PC crystals predominantly appear in three morphologies, hexagonal, needle-like and feather-like, as shown in Fig. 2. Different crystallization screens favour certain combinations of morphologies and sizes, but there is no direct correlation of the precipitant, $\mathrm{pH}$ value or additives that results in certain morphologies. 
Table 4

Selected examples of the diversity of crystallization conditions and indexing parameters for data collected from crystals larger than $70 \mu \mathrm{m}$.

The space group is $R 32$ and the unit-cell parameters are $a=b=187, c=60 \AA$, $\alpha=\beta=90, \gamma=120^{\circ}$. The complete results are given in Supplementary Tables $\mathrm{S} 2$ and S3. The protein buffer is $20 \mathrm{~m} M$ Tris $\mathrm{pH} 8.0,100 \mathrm{~m} M \mathrm{NaCl}$.

\begin{tabular}{|c|c|c|}
\hline Crystallization solution & $\mathrm{pH}$ & $\begin{array}{l}\text { Maximum } \\
\text { resolution } \\
(\AA)\end{array}$ \\
\hline $\begin{array}{l}1.5 \% \text { vitamins mix, } 0.1 M \text { imidazole, MES, } 10 \% \text { MPD, } \\
10 \% \text { PEG } 1000,10 \% \text { PEG } 3350\end{array}$ & 6.5 & 1.05 \\
\hline $2.0 \mathrm{M}$ ammonium sulfate, $0.1 \mathrm{M}$ sodium acetate & 4.6 & 1.10 \\
\hline $0.1 M$ Bicine, $10 \%(w / v)$ PEG 6000 & 9 & 1.10 \\
\hline $\begin{array}{l}0.1 M \text { ammonium sulfate, } 0.3 M \text { sodium formate, } 0.1 M \text { Tris, } \\
3 \%(w / v) \gamma \text {-PGA }\left(\mathrm{Na}^{+} \text {form, LM), } 10 \%(w / v) \text { PEG } 2000\right. \\
\text { MME }\end{array}$ & 7.8 & 1.12 \\
\hline $\begin{array}{l}0.3 M \text { potassium bromide, } 0.1 M \text { sodium acetate, } 8 \%(w / v) \\
\gamma \text {-PGA }\left(\mathrm{Na}^{+} \text {form, } \mathrm{LM}\right)\end{array}$ & 5 & 1.16 \\
\hline $40 \%$ PEG $300,100 \mathrm{~m} M$ phosphate-citrate & 4.5 & 1.18 \\
\hline $\begin{array}{l}0.2 M \text { magnesium chloride hexahydrate, } 0.1 M \mathrm{MES}, \\
14 \%(v / v) \text { pentaerythritol propoxylate }(17 / 8 \mathrm{PO} / \mathrm{OH})\end{array}$ & 5.5 & 1.19 \\
\hline $\begin{array}{l}0.2 \mathrm{M} \text { potassium citrate tribasic monohydrate, } 15 \%(w / v) \\
\text { Sokalan } \mathrm{CP} 42\end{array}$ & - & 1.22 \\
\hline $\begin{array}{l}0.1 M \text { MES, } 30 \%(w / v) \text { poly (acrylic acid sodium salt) } 5100 \text {, } \\
10 \% \text { ethanol }\end{array}$ & 6 & 1.25 \\
\hline $\begin{array}{l}0.2 M \text { zinc acetate dihydrate, } 0.1 M \text { sodium acetate, } \\
10 \%(w / v) \text { PEG } 3000\end{array}$ & 4.5 & 1.26 \\
\hline $\begin{array}{l}0.1 M \text { Tris, } 5 \%(w / v) \gamma \text {-PGA }\left(\mathrm{Na}^{+} \text {form, LM }\right), 20 \%(w / v) \\
\text { PEG } 3350\end{array}$ & 7.8 & 1.26 \\
\hline $\begin{array}{l}0.2 M \text { sodium chloride, } 0.1 M \text { Bicine, } 20 \%(w / v) \\
\text { poly(acrylic acid sodium salt) } 2100\end{array}$ & 9 & 1.37 \\
\hline $0.1 M$ MES, $12 \%$ polyvinylpyrrolidone & 5.5 & 1.54 \\
\hline
\end{tabular}

Table 5

Summary of the results from the X-ray diffraction data collected from single crystals.

For C-PC at $\mathrm{pH} 6.5,58$ data sets were analyzed and for C_PC at $\mathrm{pH} 8.0$, there were 60 data sets. The complete results are given in Supplementary Tables S2 and S3.

\begin{tabular}{lll}
\hline & \multicolumn{2}{l}{ No. of data sets } \\
\cline { 2 - 3 } Data collection/indexing & C-PC at pH 6.5 & C-PC at pH 8.0 \\
\hline Resolution $<1.2 \AA$ & $17 / 58$ & $24 / 60$ \\
Resolution $<1.35 \AA$ & $28 / 58$ & $36 / 60$ \\
Resolution $<2 \AA$ & $53 / 58$ & $53 / 60$ \\
Space group $R 32$ & $53 / 58$ & $47 / 60$ \\
Space group $P 6_{3}$ & $3 / 58$ & $11 / 60$ \\
Other space groups & $3 / 58$ & $2 / 60$ \\
\hline
\end{tabular}

It is worth mentioning that 41 individual crystals diffracted to a resolution of higher than $1.2 \AA$, which improves the highest maximum resolution of a cyanobacterial C-phycocyanin structure reported to date, which is $1.35 \AA$ (PDB entry 3o18; David et al., 2011). More than $70 \%$ of all data collected extended to a resolution of better than $1.5 \AA$, and $95 \%$ extended to better than $2 \AA$ resolution (Supplementary Fig. S6). This outcome is very likely to be owing to the high purity of C-PC produced using the modified purification method (see Section 2 and Fig. 1).

Analysis of these results shows that C-PC assembly is not affected by the crystallization conditions. The protein crystallizes in a particular space group independent of the $\mathrm{pH}$ value or the precipitants, whether for example high salt, PEG or ethanol are present (examples are provided in Table 4 and
Supplementary Tables S2 and S3). This effect was previously concealed and, remarkably, the tendency of C-PC to preferentially crystallize in space group $R 32$ is unexpected. Compared with well characterized crystal model systems, such as hen egg-white lysozyme, for which a vast number of symmetries have been reported, C-PC prefers to crystallize with $R 32$ symmetry. As mentioned earlier, the purified protein can self-assemble into nanosized structures in solution. This effect is very likely to promote crystal nucleation and to act as a seed during crystal growth. Since phycobilosomes are naturally organized in the cells (Blankenship, 2015), the in vivo C-PC assembly exhibits a tricylindrical core, from which six rods composed of three $\mathrm{PC}$ hexamers radiate, in order to assemble superior rigid antenna-like structures to expand light harvesting (Wang \& Moerner, 2015).

\subsection{Crystal packing and symmetries}

Amongst the diffraction data sets that were collected and analysed, there are distinct variations in the crystal packing and the symmetry. As summarized in Table 5, for the majority of the data that were collected and analysed, C-PC crystallizes in high-symmetry space groups. More precisely, there are four different space groups. The majority of all crystals belong to the rhombohedral space group R32 (Fig. 4). The hexagonal space group was also found, with two different unit cells, referred to as $P 6_{3}$-large and $P 6_{3}$-small (Figs. 5 and 6), as was the orthorhombic space group $P 2_{1} 2_{1} 2$ (Fig. 7). The latter was only observed once among the data sets collected. The crystallization conditions for each of the data sets are given in Supplementary Tables S2 and S3. Interestingly, the addition of small drug-like molecules such as cholic acid derivatives, an anaesthetic alkaloids mixture or amino acids (Gorrec, 2009, 2015; Blundell, 2017) led to the formation of new crystal packing and novel high-resolution crystal structures of C-PC, as shown and explained in Figs. 5, 6 and 7.

The crystal contacts in $P 6_{3}$-small are solely mediated between the $\alpha$-subunits along the $a b$ unit-cell plane and are arranged across the $\alpha$ - and $\beta$-subunits along the $c$ axis, as shown in Fig. 5(a). Interestingly, the packing along the latter axis is also supported by a ligand, tetracaine, which was picked up from the crystallization conditions. It binds mostly via water-mediated hydrogen bonds in the cleft between the $\alpha$ and $\beta$-units of the two molecular crystal planes. This particular molecule allows the formation of this specific packing as it occupies the binding site, enabling, for instance, the assembly of the dodecameric, doughnut-shaped structure that is observed in the rhombohedral and orthorhombic space groups (Fig. 5a). This packing allows the generation of a solvent channel spanning the protein crystal. A loop region (Fig. 5b) responsible for binding one of the covalent cofactors and another moderately rigid structure contributes to the formation of this approximately $20 \AA$ wide pore (Figs. $5 c$ and $5 d$ ).

In contrast to the $P 6_{3}$-small unit cell, the arrangement of the molecules is slightly altered within the $P 6_{3}$-large unit cell. The tetrameric ring structure remains the same as a building block, but the packing is different (Figs. $5 c$ and $6 a$ ). The crystal 
contacts are mediated by both the $\alpha$ - and $\beta$-subunits in each crystal lattice direction (Fig. $6 d$ ). The linearly stacked rings form a tube-like structure (Figs. $6 c$ and $6 d$ ). The open, accessible solvent channels have a diameter of about $70 \AA$ and permit the diffusion of average-sized macromolecules such as peptides throughout the crystal (Erickson, 2009; Lukatsky \& Shakhnovich, 2008), as depicted in Figs. 6(a) and 6(c). In the orthorhombic space group $P 2{ }_{1} 2_{1} 2$ (Fig. 7) the $\alpha$-subunits solely mediate crystal contacts within the $a b$ plane, and the $\beta$-subunit connects the doughnut-shaped rings in the $c$ direction.

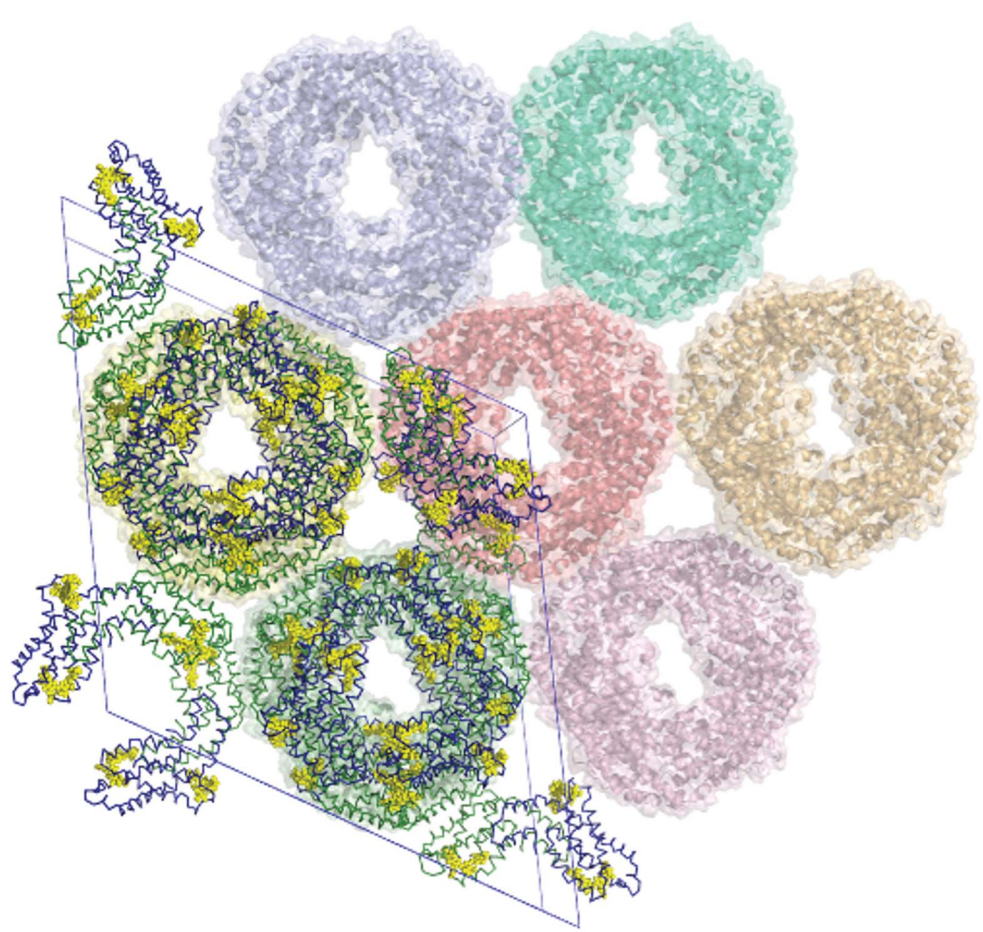

(a)

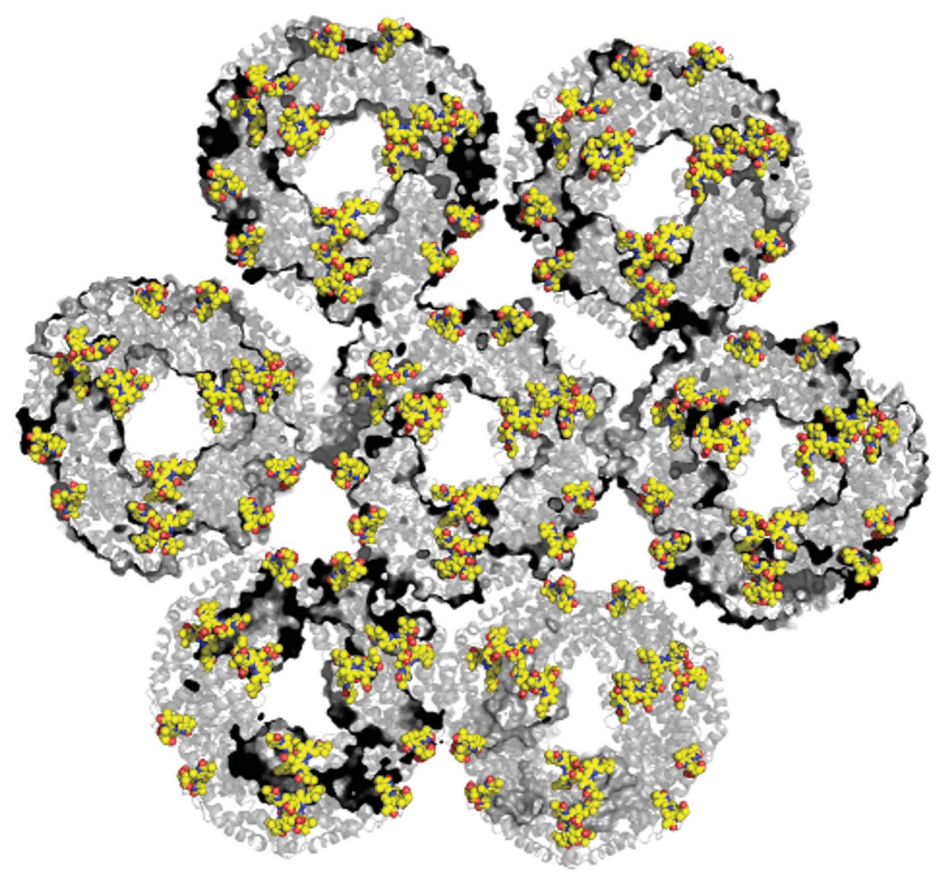

(c)

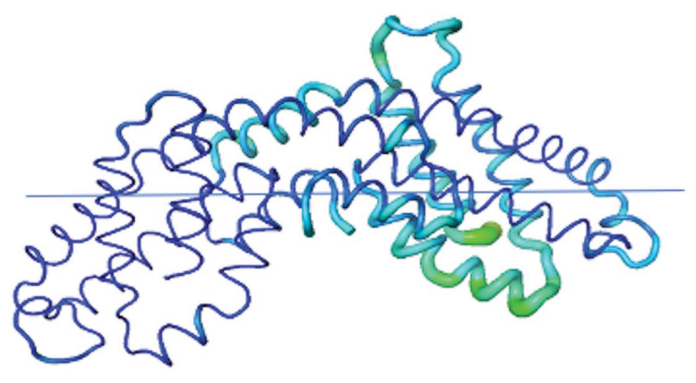

$\int 90^{\circ}$

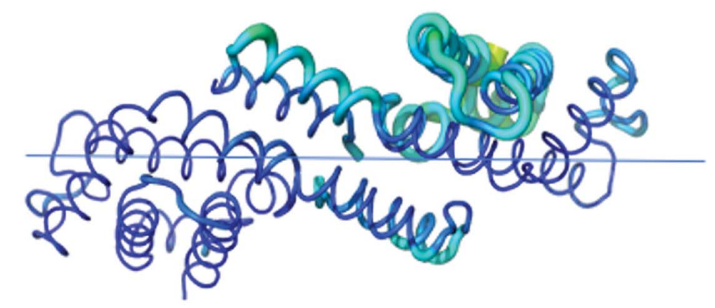

(b)
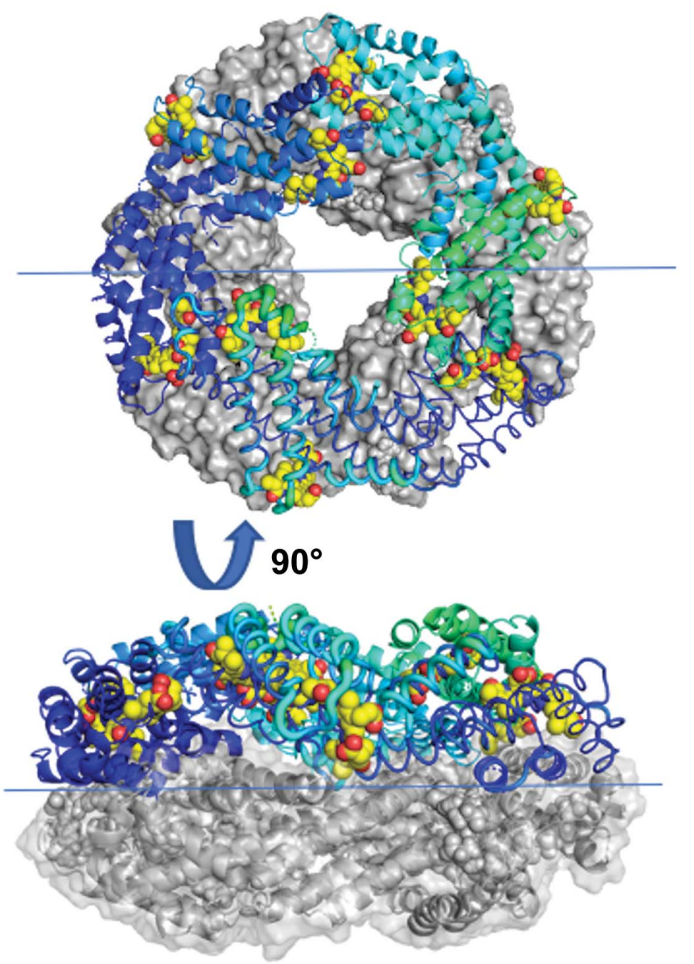

(d)

Figure 4

Crystal packing and structural details of C-PC crystallized in the rhombohedral space group $R 32$ (data set 10, Supplementary Table S3). (a) The unit-cell content is depicted as an overlay in the crystal-packing context. (b) $B$-factor plot as a function of position in the individual heterodimeric C-PC molecules. (c) Each heterodimeric C-PC molecule harbours three attached phycocyanobilin ligands (yellow). (d) The dodecameric ring structure with the top ring in cartoon representation and the bottom ring as a surface (grey). 
As discussed above, the addition of small molecules to the crystallization experiments creates different symmetries. The addition of an anaesthetic alkaloids mixture reshaped the pattern of preferred crystal contacts and thereby altered the crystal packing. As a result, two distinct unit cells in space group $P 6_{3}$ can be recognized, with the additive molecule clearly visible in the electron density of the $P 6_{3}$-small structure.

Analysis of the crystal packing in both hexameric space groups reveals a ring structure. As shown in Figs. 5 and 6, this is not surprising owing to the nature of the phycobilosomes and previously reported phycocyanin structures (Supplementary Table S1). In detail, the crystal interfaces between the staggered rings cover 5000 and $7180 \AA^{2}$, with a buried-toexposed surface ratio of 0.3 (Fig. 5 and Supplementary Fig. S8). These interfaces are specific and contribute about $50 \mathrm{kcal} \mathrm{mol}^{-1}$ per interaction and might be one driving force for this type of crystal packing. This denser packing is also reflected in the increased buried surface area as compared using the regular hexagonal building block (Supplementary Fig. S8, Fig. 8).

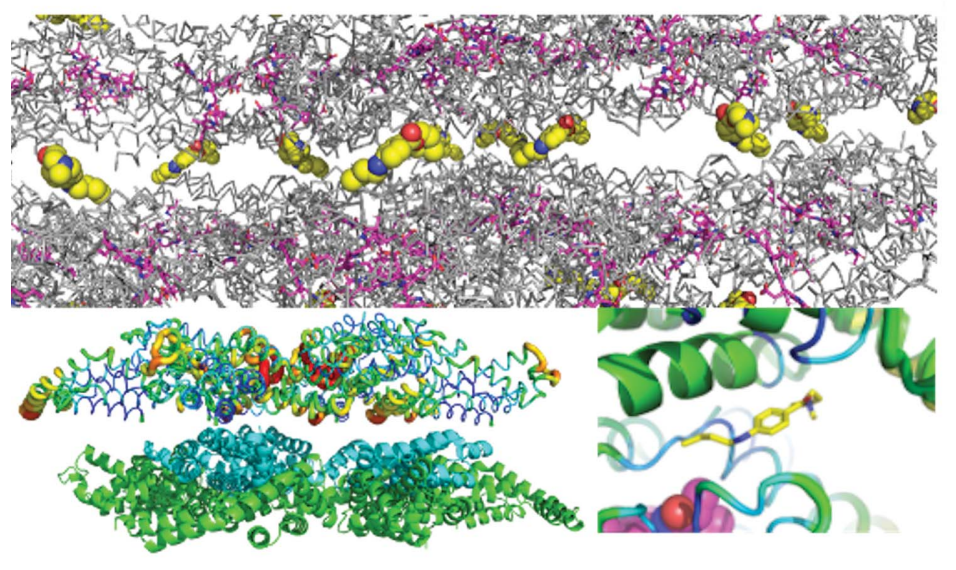

(a)

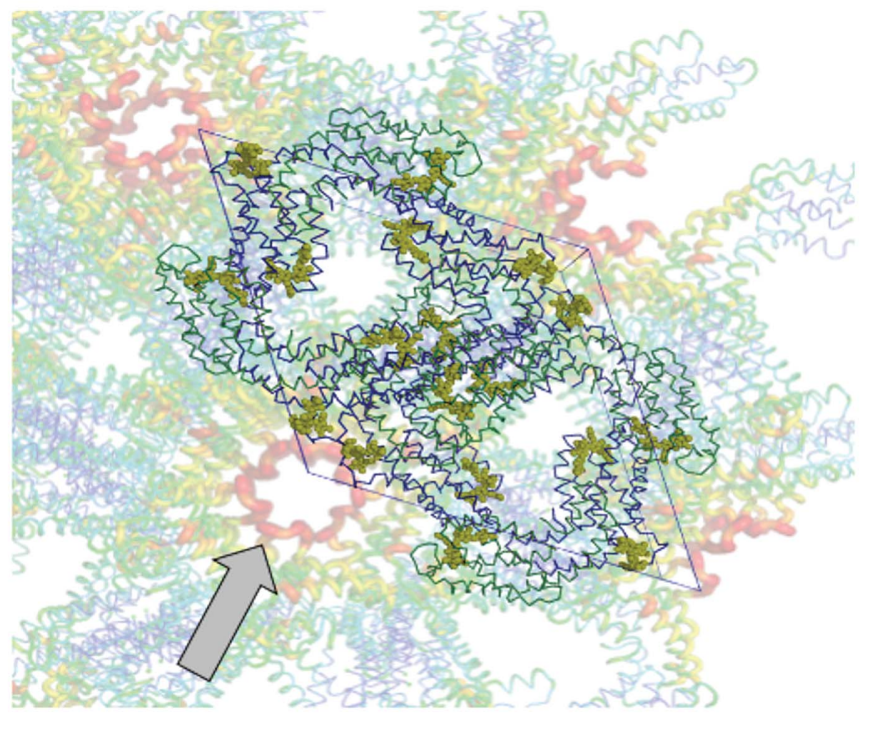

(c)

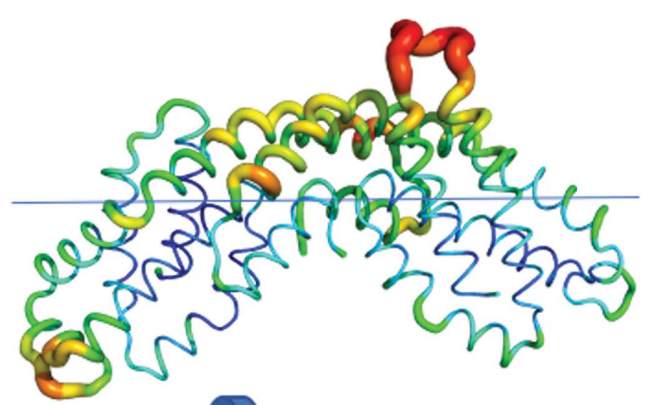

$190^{\circ}$

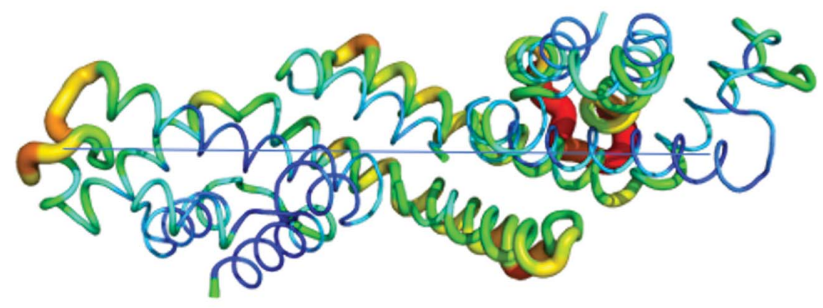

(b)

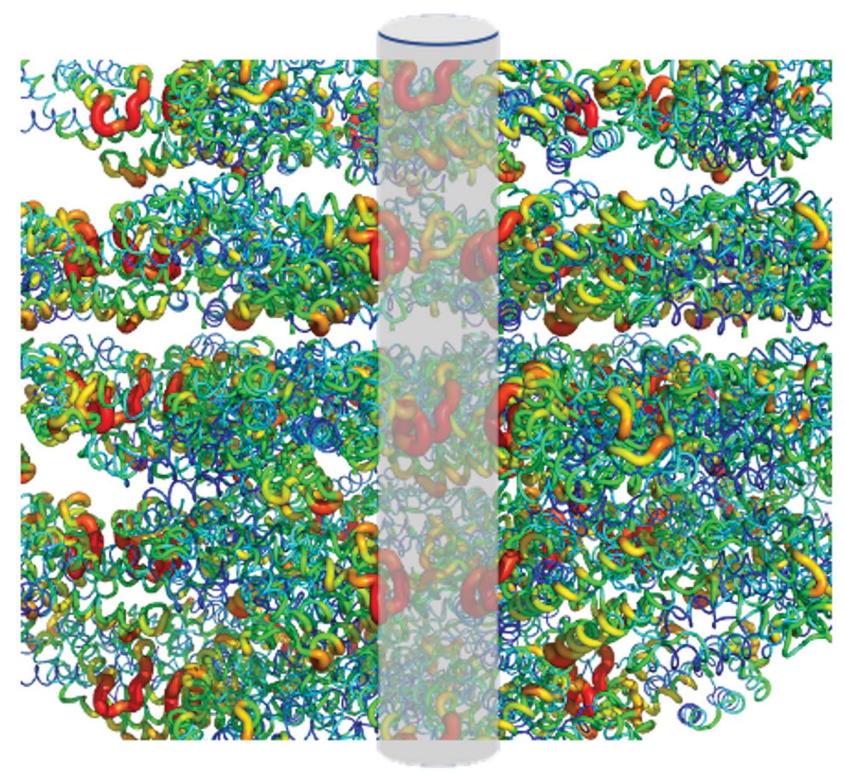

(d)

Figure 5

C-PC in the hexagonal space group $P 6_{3}$ (data set 42, Supplementary Table S2). (a) Crystal contacts and the layered hexameric ring structure (coloured grey) are mediated by the additive molecule tetracaine (coloured yellow). (b) The $B$ factors are plotted onto the heterodimeric structure. (c) The unitcell content is superposed onto the crystal packing coloured according to $B$ factors. The arrow points to the small pore flanked by the loop region binding one of the cofactors, coloured dark yellow. $(d)$ The tunnel spans across the crystal. Individual layers are coloured according to the $B$ factors and the tunnel is depicted in light grey. 
In contrast, the structures determined in the rhombohedral and orthorhombic space groups compose a stable dodecameric molecule. Two hexameric rings associate turned towards each other into a doughnut-shaped superstructure. The interaction surface area covers more than $61000 \AA^{2}$, with a ratio of surface-exposed versus buried area of almost 1 (Fig. 6 and Supplementary Fig. S8). The free energy for the assembly is calculated to be approximately $500 \mathrm{kcal} \mathrm{mol}^{-1}$ and indicates another driving force for this large stable tertiary assembly (Supplementary Fig. S7). This superstructure has not been seen in the crystal packing of any C-PC and is very likely to be connected to the natural assembly of the phycobilosome rods, although this assumption needs further in-depth investigation using other methods.
The conventionally quantified ratio of buried and solventaccessible exposed surface area (ASA; Lee \& Richards, 1971) showed a significant difference amongst the C-PC protein structures (Fig. 8). The ASA ratio in these assemblies increases, which is also a driving force in addition to the large gain in solvation free-energy gain upon the arrangement of these larger molecular structures (Fig. 8 and Supplementary Fig. S8). The specific packing of the C-PC molecules in each case might be correlated with the natural activity of C-PC as a light-harvesting antenna. Similarly, other proteins, for example chaperones or crystallines, alter their oligomeric state depending on their role in activity and their physiological environment (Jaenicke, 1996; Fu et al., 2003; Libonati \& Gotte, 2004).

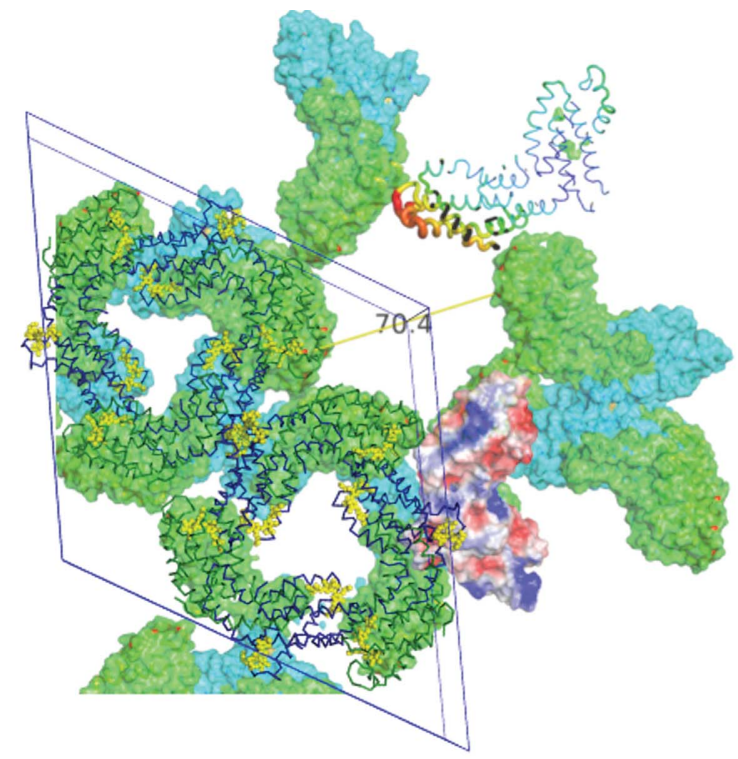

(a)

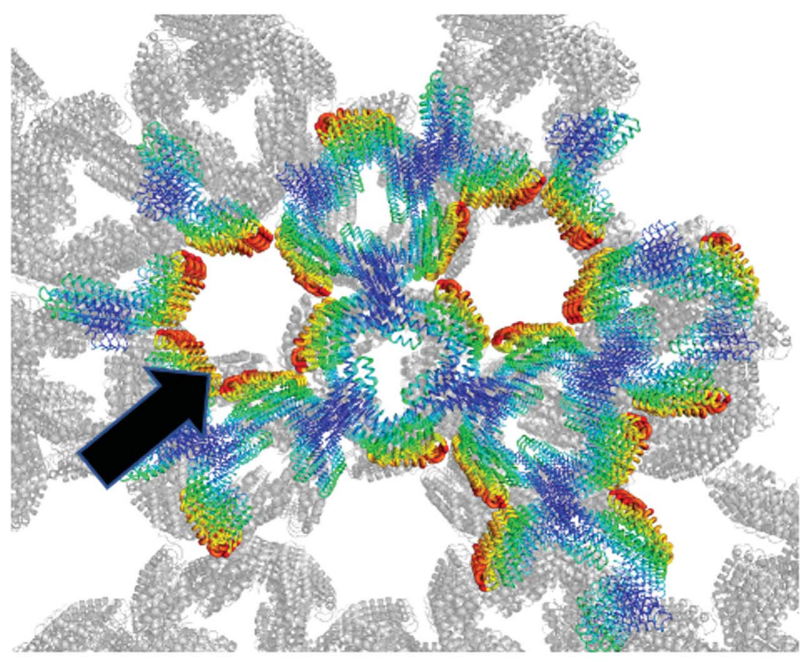

(c)
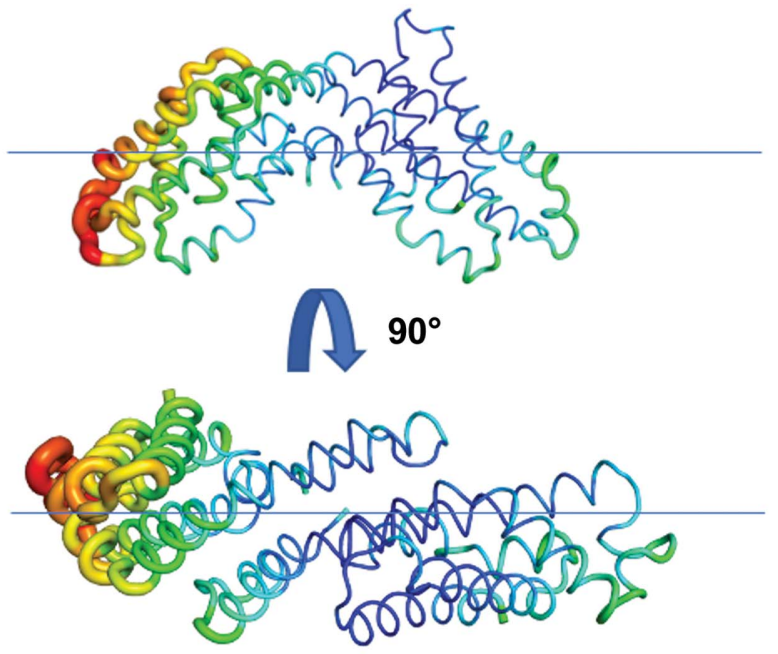

(b)

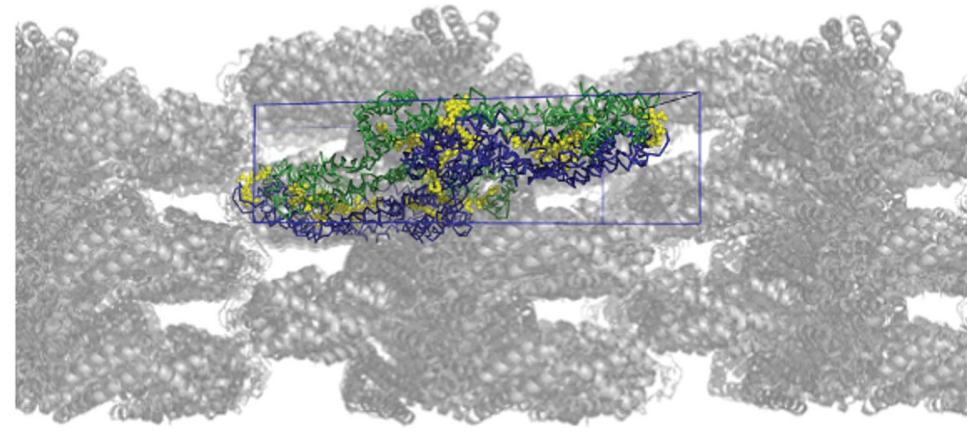

(d)

Figure 6

C-PC structures crystallized in space group $\mathrm{Pb}_{3}$ with large unit-cell dimensions (data set 48 , Supplementary Table S2). (a) The unit-cell content is indicated by the ribbon model facing the $a b$ plane. The opening of the solvent channel was calculated and is indicated in $\AA$. $(b)$ The temperature factors are mapped onto an individual heterodimeric C-PC protein molecule. (c) Flexible parts flank the open solvent channel and the black arrow indicates the solvent channel with about $70 \AA$ diameter. $(d)$ Protein packing along the $a c$ axis. The individual C-PC monomers are colour-coded blue and green. The covalently bound ligands are shown in yellow. 

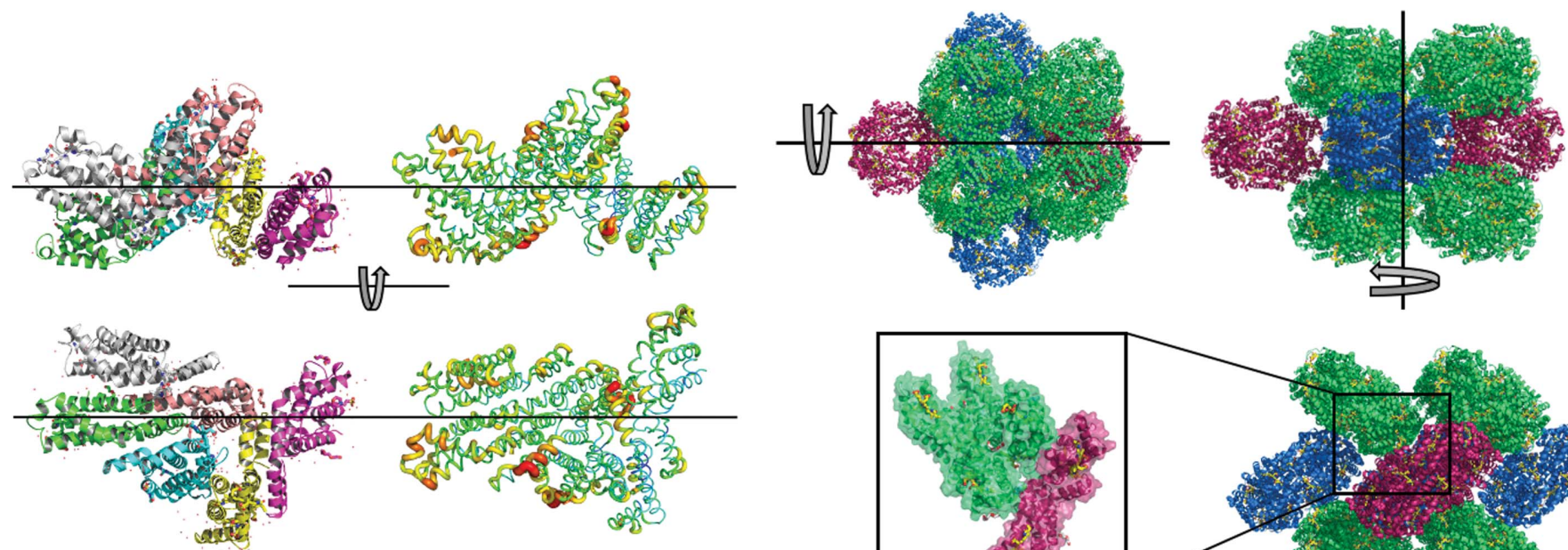

$(a)$
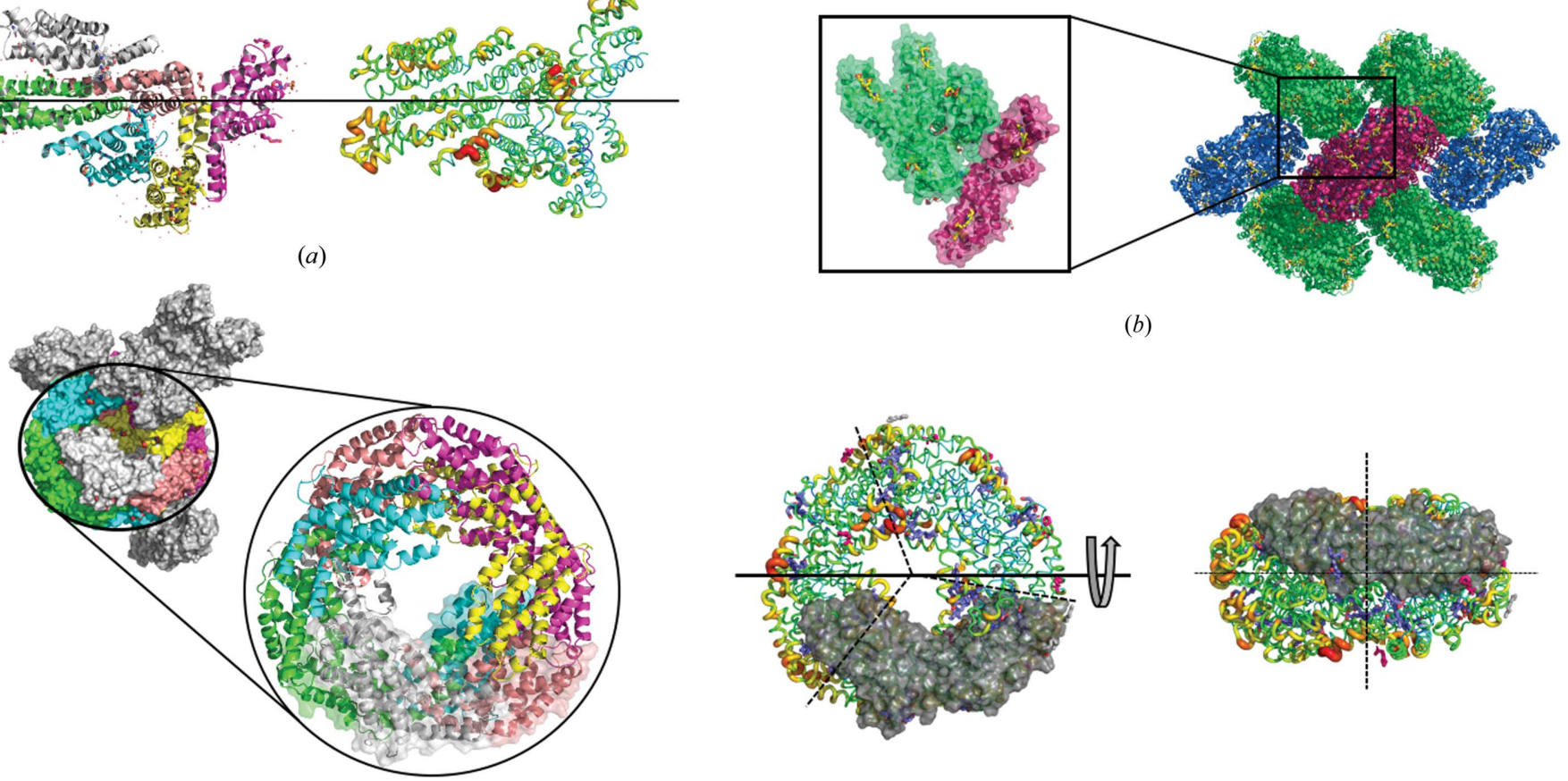

(c)

(b)
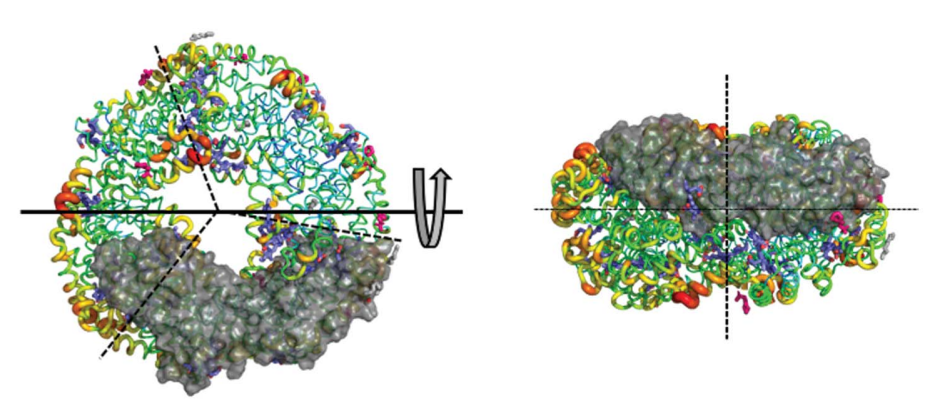

Figure 7

Crystal packing in the orthorhombic space group (data set 56, Supplementary Table S3). (a) The content of the asymmetric unit is depicted with each chain coloured individually (left) and coloured according to the determined temperature factors (right). (b) Six heterodimeric molecules assemble the doughnut-shaped structure, with individual colours indicating the three angles. The content of the asymmetric unit is magnified. $(c)$ The content of the unit cell contains one of the dodecameric rings, and its position within the molecular arrangement is indicated. $(d)$ The $120^{\circ}$ symmetry of the ring structure is indicated by the dashed lines in the left panel. The chains are coloured according to their $B$ factors, with one heterodimeric C-PC molecule shown in surface representation.

In order to examine any structural changes in the area of the phycocyanobilin, we compared the region of the blue chromophore in all of the structures (Supplementary Table $\mathrm{S} 4)$. In the small hexagonal space group $\left(P 6_{3}\right.$-small), the molecular assembly of the two chains is kinked by about $6^{\circ}$ from the chains of the structural models and the cofactor position differs by $2.8 \AA$ from that in the other three structure (labelled 2 in Supplementary Figs. S9b and S9c). Despite this minor difference, the overall position of phycocyanobilin and the orientation of the intrinsic ligands to the respective hosts is similar in all models.

Summarizing, C-PC crystallizes in a vast number of significantly different conditions. The addition of small molecules, termed additives, gives rise to the variety of observed crystal packings. Despite the different symmetry, no notable modulation of the protein structures could be detected. This is in agreement with the observations reported for other proteins, in which changes in their oligomerization state are correlated with their activity (Jiang et al., 2008).
In conclusion, the mechanisms of protein crystal packing are complex and unclear, and the effects of additives are generally not well understood (Luo et al., 2018; Carugo et al., 2017). In this study, we discuss the unique crystallization behaviour of C-phycocyanin, which includes effortless highquality crystal formation with the majority of available crystallization precipitants. The effect of additives and the variation of crystal packing offers a simple new system for future in-depth investigations of protein crystallization mechanisms.

\section{Applications and outlook}

C-phycocyanin easily produces well diffracting crystals with many morphologies, sizes and symmetries.

The molecular packing within the large $P 6_{3}$ unit cell, with large and open solvent channels extending over the crystalline material, could become a scaffold to accompany foreign protein molecules and facilitate the accommodation of passenger proteins in pores (Fig. 7). Consequently, crystals in 


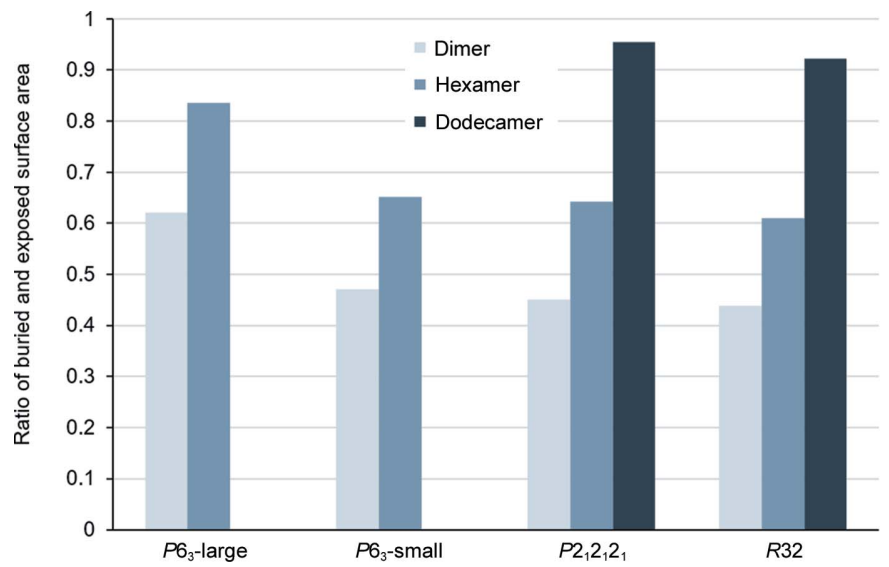

Figure 8

The heterodimeric building blocks assemble into higher oligomeric structures. The ratios of buried and surface-exposed areas have been calculated and are plotted for all space groups and crystal packings (Krissinel \& Henrick, 2007). Values for the dimer correspond to the assembled heterodimeric $\alpha \beta$ chain. The hexamer comprises an assembly of three heterodimers and the dodecamer is the association of two of these six-membered rings.

this space group may become an additional tool for studying the existing scaffolds of highly porous protein crystals (Stura et al., 2002; Kowalski et al., 2019).

Protein crystals are routinely used, for example in technical beam alignment, detector calibration, the delivery and injection testing of crystal suspensions, investigation of the crystallization process, phasing techniques, analysing protein dynamics on a short time scale, for educational purposes and more (Haas, 2020; Yip \& Ward, 1996; Norrman et al., 2006; Olieric et al., 2007). Most of the proteins used for methods development in protein crystallography research are available for purchase in large amounts produced from animals, unless produced recombinantly (Kim et al., 2019). C-PC is produced by cyanobacteria, which grow more rapidly and are less nutritionally demanding (Yu et al., 2015), and is purified using a one-column purification protocol. As one of many valuable biomolecules produced with a minimal amount of waste, it can provide crystallographers with an excellent multipurpose sample with possibilities to modify the molecular packing of crystals.

Finally, we suggest that C-PC may have applications in intermolecular cross-linking, a variety of assays and also in passenger 'guest' molecule imaging (Snapp, 2005). The rigid compact $\alpha$-helical folding and crystal lattice may stabilize otherwise unstable proteins or support the accommodation of small molecules (Vyncke et al., 2019; Ernst et al., 2019). The advantageous naturally bright colour and intrinsic fluorescence make the unambiguous identification of C-PC protein crystals very convenient, particularly in experiments utilizing and scoring microcrystals (Meents et al., 2017).

\section{Related literature}

The following references are cited in the supporting information for this article: Lieske et al. (2019).

\section{Acknowledgements}

We acknowledge DESY (Hamburg, Germany), a member of the Helmholtz Association HGF. Parts of this research were carried out on beamline P11 of the PETRA III facility. Open access funding enabled and organized by Projekt DEAL.

\section{References}

Adir, N., Vainer, R. \& Lerner, N. (2002). Biochim. Biophys. Acta, 1556, 168-174.

Blankenship, R. E. (2015). Proc. Natl Acad. Sci. USA, 112, 1375113752.

Blundell, T. L. (2017). IUCrJ, 4, 308-321.

Boyd, R. W. (2008). Nonlinear Optics, 3rd ed., pp. 1-67. New York: Academic Press.

Carugo, O., Blatova, O. A., Medrish, E. O., Blatov, V. A. \& Proserpio, D. M. (2017). Sci. Rep. 7, 13209.

Chayen, N. E. (2009). Adv. Protein Chem. Struct. Biol. 77, 1-22.

Chayen, N. E. \& Saridakis, E. (2008). Nat. Methods, 5, 147-153.

Chayen, N. E., Shaw Stewart, P. D. \& Blow, D. M. (1992). J. Cryst. Growth, 122, 176-180.

David, L., Marx, A. \& Adir, N. (2011). J. Mol. Biol. 405, 201-213.

DePristo, M. A., de Bakker, P. I. W. \& Blundell, T. L. (2004). Structure, 12, 831-838.

Emsley, P., Lohkamp, B., Scott, W. G. \& Cowtan, K. (2010). Acta Cryst. D66, 486-501.

Erickson, H. P. (2009). Biol. Proced. Online, 11, 32-51.

Ernst, P., Plückthun, A. \& Mittl, P. R. E. (2019). Sci. Rep. 9, 15199.

Falke, S. \& Betzel, C. (2019). Radiation in Bioanalysis: Spectroscopic Techniques and Theoretical Methods, edited by A. S. Pereira, P. Tavares \& P. Limão-Vieira, pp. 173-193. Cham: Springer Nature Switzerland.

Feiler, C. G., Wallacher, D. \& Weiss, M. S. (2019). J. Vis. Exp., e59722.

Frey, M., Genovesio-Taverne, J.-C. \& Fontecilla-Camps, J. C. (1991). J. Phys. D Appl. Phys. 24, 105-110.

Fu, X., Liu, C., Liu, Y., Feng, X., Gu, L., Chen, X. \& Chang, Z. (2003). Biochem. Biophys. Res. Commun. 310, 412-420.

Gorrec, F. (2009). J. Appl. Cryst. 42, 1035-1042.

Gorrec, F. (2015). Acta Cryst. F71, 831-837.

Groot, B. L. de, Hayward, S., van Aalten, D. M. F., Amadei, A. \& Berendsen, H. J. C. (1998). Proteins, 31, 116-127.

Haas, D. J. (2020). IUCrJ, 7, 148-157.

He, H., Chen, L., Wang, Z., Zhang, L., Ge, T., Xiang, X., Wang, S., Huang, Y. \& Li, S. (2020). Cryst. Growth Des. 20, 6877-6887.

Jaenicke, R. (1996). FASEB J. 10, 84-92.

Jiang, J., Zhang, X., Chen, Y., Wu, Y., Zhou, Z. H., Chang, Z. \& Sui, S.-F. (2008). Proc. Natl Acad. Sci. USA, 105, 11939-11944.

Karplus, P. A. \& Diederichs, K. (2012). Science, 336, 1030-1033.

Kim, S. W., Less, J. F., Wang, L., Yan, T., Kiron, V., Kaushik, S. J. \& Lei, X. G. (2019). Annu. Rev. Anim. Biosci. 7, 221-243.

Kowalski, A. E., Johnson, L. B., Dierl, H. K., Park, S., Huber, T. R. \& Snow, C. D. (2019). Biomater. Sci. 7, 1898-1904.

Krissinel, E. \& Henrick, K. (2007). J. Mol. Biol. 372, 774-797.

Lee, B. \& Richards, F. M. (1971). J. Mol. Biol. 55, 379-400.

Libonati, M. \& Gotte, G. (2004). Biochem. J. 380, 311-327.

Liebschner, D. (2018). Acta Cryst. F74, 74-75.

Liebschner, D., Afonine, P. V., Baker, M. L., Bunkóczi, G., Chen, V. B., Croll, T. I., Hintze, B., Hung, L.-W., Jain, S., McCoy, A. J., Moriarty, N. W., Oeffner, R. D., Poon, B. K., Prisant, M. G., Read, R. J., Richardson, J. S., Richardson, D. C., Sammito, M. D., Sobolev, O. V., Stockwell, D. H., Terwilliger, T. C., Urzhumtsev, A. G., Videau, L. L., Williams, C. J. \& Adams, P. D. (2019). Acta Cryst. D75, 861-877.

Lieske, J., Cerv, M., Kreida, S., Komadina, D., Fischer, J., Barthelmess, M., Fischer, P., Pakendorf, T., Yefanov, O., Mariani, V., Seine, T., Ross, B. H., Crosas, E., Lorbeer, O., Burkhardt, A., Lane, T. J., 
Guenther, S., Bergtholdt, J., Schoen, S., Törnroth-Horsefield, S., Chapman, H. N. \& Meents, A. (2019). IUCrJ, 6, 714-728.

Lorber, B. (2005). Cryst. Growth Des. 5, 17-19.

Lukatsky, D. B. \& Shakhnovich, E. I. (2008). Phys. Rev. E, 77, 020901. Luo, Y., Na, Z. \& Slavoff, S. A. (2018). Biochemistry, 57, 2424-2431. McPherson, A. \& Cudney, B. (2006). J. Struct. Biol. 156, 387-406.

Meents, A., Wiedorn, M. O., Srajer, V., Henning, R., Sarrou, I., Bergtholdt, J., Barthelmess, M., Reinke, P. Y. A., Dierksmeyer, D., Tolstikova, A., Schaible, S., Messerschmidt, M., Ogata, C. M., Kissick, D. J., Taft, M. H., Manstein, D. J., Lieske, J., Oberthuer, D., Fischetti, R. F. \& Chapman, H. N. (2017). Nat. Commun. 8, 1281.

Nield, J., Rizkallah, P. J., Barber, J. \& Chayen, N. E. (2003). J. Struct. Biol. 141, 149-155.

Norrman, M., Ståhl, K., Schluckebier, G. \& Al-Karadaghi, S. (2006). J. Appl. Cryst. 39, 391-400.

Olieric, V., Schreiber, A., Lorber, B. \& Pütz, J. (2007). Biochem. Mol. Biol. Educ. 35, 280-286.

Panjikar, S., Thomsen, L., O'Donnell, K. M. \& Riboldi-Tunnicliffe, A. (2015). J. Appl. Cryst. 48, 913-916.

Rupp, B. (2010). Biomolecular Crystallography: Principles, Practice, and Application to Structural Biology. New York: Garland Science.
Sauter, A., Roosen-Runge, F., Zhang, F., Lotze, G., Feoktystov, A., Jacobs, R. M. J. \& Schreiber, F. (2015). Faraday Discuss. 179, 41-58. Snapp, E. (2005). Curr. Protoc. Cell Biol. 27, 21.4.1-21.4.13.

Sparta, K. M., Krug, M., Heinemann, U., Mueller, U. \& Weiss, M. S. (2016). J. Appl. Cryst. 49, 1085-1092.

Spence, J. C. H. (2017). IUCrJ, 4, 322-339.

Stura, E. A., Taussig, M. J., Sutton, B. J., Duquerroy, S., Bressanelli, S., Minson, A. C. \& Rey, F. A. (2002). Acta Cryst. D58, 1715-1721.

Vyncke, L., Masschaele, D., Tavernier, J. \& Peelman, F. (2019). Int. J. Mol. Sci. 20, 2058.

Wang, Q. \& Moerner, W. E. (2015). Proc. Natl Acad. Sci. USA, 112, 13880-13885.

Winn, M. D., Ballard, C. C., Cowtan, K. D., Dodson, E. J., Emsley, P., Evans, P. R., Keegan, R. M., Krissinel, E. B., Leslie, A. G. W., McCoy, A., McNicholas, S. J., Murshudov, G. N., Pannu, N. S., Potterton, E. A., Powell, H. R., Read, R. J., Vagin, A. \& Wilson, K. S. (2011). Acta Cryst. D67, 235-242.

Yip, C. M. \& Ward, M. D. (1996). Biophys. J. 71, 1071-1078.

Yu, J., Liberton, M., Cliften, P. F., Head, R. D., Jacobs, J. M., Smith, R. D., Koppenaal, D. W., Brand, J. J. \& Pakrasi, H. B. (2015). Sci. Rep. 5, 8132. 